\title{
GAMMA STABILITY IN FREE PRODUCT VON NEUMANN ALGEBRAS
}

\author{
CYRIL HOUDAYER
}

\begin{abstract}
Let $(M, \varphi)=\left(M_{1}, \varphi_{1}\right) *\left(M_{2}, \varphi_{2}\right)$ be a free product of arbitrary von Neumann algebras endowed with faithful normal states. Assume that the centralizer $M_{1}^{\varphi_{1}}$ is diffuse. We first show that any intermediate subalgebra $M_{1} \subset Q \subset M$ which has nontrivial central sequences in $M$ is necessarily equal to $M_{1}$. Then we obtain a general structural result for all the intermediate subalgebras $M_{1} \subset Q \subset M$ with expectation. We deduce that any diffuse amenable von Neumann algebra can be concretely realized as a maximal amenable subalgebra with expectation inside a full nonamenable type $\mathrm{III}_{1}$ factor. This provides the first class of concrete maximal amenable subalgebras in the framework of type III factors. We finally strengthen all these results in the case of tracial free product von Neumann algebras.
\end{abstract}

\section{Introduction AND StATEMENT OF the MAIn RESUlts}

A von Neumann algebra $M \subset \mathbf{B}(H)$ (with separable predual) is amenable if there exists a norm one projection $E: \mathbf{B}(H) \rightarrow M$. By Connes' celebrated result Co75b, all the amenable von Neumann algebras are hyperfinite. Moreover, the amenable or hyperfinite factors are completely classified by their flows of weights (see Co72, Co75b, Co85, Ha84]). In particular, there is a unique amenable $\mathrm{II}_{1}$ factor [Co75b]: it is the hyperfinite $\mathrm{II}_{1}$ factor of Murray and von Neumann MvN43.

Since the amenable von Neumann algebras form a monotone class, any von Neumann algebra admits maximal amenable subalgebras. The first concrete examples of maximal amenable subalgebras inside $\mathrm{II}_{1}$ factors were obtained by Popa in Po83. He showed that any generator masa $A$ in a free group factor $\mathrm{L}\left(\mathbf{F}_{n}\right)$ with $n \geq 2$ is maximal amenable. This result answered in the negative a question raised by Kadison. Indeed, $A \subset \mathrm{L}\left(\mathbf{F}_{n}\right)$ is an abelian subalgebra generated by a selfadjoint operator and yet there is no intermediate hyperfinite subfactor in $\mathrm{L}\left(\mathbf{F}_{n}\right)$ which contains $A$ as a subalgebra. Popa discovered in $\mathrm{Po} 83$ a powerful method to prove that a given amenable subalgebra is maximal amenable inside an ambient $\mathrm{II}_{1}$ factor. Using this strategy for the generator masa $A \subset \mathrm{L}\left(\mathbf{F}_{n}\right)$, he first showed that $A$ satisfies a certain asymptotic orthogonality property and then deduced that $A$ is maximal amenable in $\mathrm{L}\left(\mathbf{F}_{n}\right)$ using various mixing techniques. His results actually showed that the generator masa $A$ is maximal Gamma inside $\mathrm{L}\left(\mathbf{F}_{n}\right)$. Recall that a $\mathrm{II}_{1}$ factor $M$ (with separable predual) has property Gamma of Murray and von Neumann MvN43] if there exists a sequence of unitaries $u_{n} \in \mathcal{U}(M)$ such that $\lim _{n \rightarrow \infty} \tau\left(u_{n}\right)=0$ and $\lim _{n \rightarrow \infty}\left\|x u_{n}-u_{n} x\right\|_{2}=0$ for all $x \in M$.

Subsequently, Cameron, Fang, Ravichandran and White proved in CFRW08 that the radial masa in a free group factor $\mathrm{L}\left(\mathbf{F}_{n}\right)$ with $2 \leq n<\infty$ is maximal amenable. Recently, the author vastly generalized in [Ho12a, Ho12b] Popa's results from [Po83] and obtained many new examples of maximal amenable subalgebras inside the crossed product $\mathrm{II}_{1}$ factors associated with free Bogoljubov actions of amenable groups. Very recently, Boutonnet and Carderi showed in $[\mathrm{BC} 13$ that any infinite maximal amenable subgroup $\Lambda$ in a Gromov word-hyperbolic group $\Gamma$ gives rise to a maximal amenable subalgebra $L(\Lambda)$ inside the group von Neumann algebra

2010 Mathematics Subject Classification. 46L10; 46L54.

Key words and phrases. Free product von Neumann algebras; Ultraproduct von Neumann algebras; Amenable von Neumann algebras; Type III factors; Property Gamma; Asymptotic orthogonality property.

Research supported by ANR grant NEUMANN. 
$\mathrm{L}(\Gamma)$. For other related results regarding maximal amenability in the framework of $\mathrm{II}_{1}$ factors, we refer the reader to [Br12, Fa06, Ga09, Ge95, Jo10, Po13, Sh05].

In this paper, we obtain new results regarding maximal amenability and Gamma stability for subalgebras of free products of arbitrary von Neumann algebras. We will be particularly interested in the structure of free product type III factors. Before stating our main results, we first introduce some terminology. Recall that a von Neumann algebra $M$ is diffuse if $M$ has no minimal projection. We say that a von Neumann subalgebra $Q \subset M$ is with expectation if there exists a faithful normal conditional expectation $E_{Q}: M \rightarrow Q$. Let now $\omega \in \beta(\mathbf{N}) \backslash \mathbf{N}$ be a non-principal ultrafilter. We say that a von Neumann algebra $M$ has property Gamma if the central sequence algebra $M^{\prime} \cap M^{\omega}$ is diffuse. Observe that in the case when $M$ is a $\mathrm{II}_{1}$ factor with separable predual, this definition is equivalent to the property Gamma of Murray and von Neumann [MvN43] (see e.g. Co74, Corollary 3.8]).

Our first main result deals with Gamma stability inside arbitrary free product von Neumann algebras $(M, \varphi)=\left(M_{1}, \varphi_{1}\right) *\left(M_{2}, \varphi_{2}\right)$. We show in Theorem \$ that the subalgebra $M_{1} \subset M$ sits in a very rigid position with respect to taking central sequences inside $M$.

Theorem A. Let $\left(M_{1}, \varphi_{1}\right)$ and $\left(M_{2}, \varphi_{2}\right)$ be $\sigma$-finite von Neumann algebras endowed with faithful normal states. Assume that the centralizer $M_{1}^{\varphi_{1}}$ is diffuse. Denote by $(M, \varphi)=$ $\left(M_{1}, \varphi_{1}\right) *\left(M_{2}, \varphi_{2}\right)$ the free product von Neumann algebra.

Then the inclusion $M_{1} \subset M$ is Gamma stable in the following sense: for every intermediate von Neumann subalgebra $M_{1} \subset Q \subset M$ such that $Q^{\prime} \cap M^{\omega}$ is diffuse, we have $Q=M_{1}$.

It is worth noticing that in the statement of Theorem $\mathrm{A}$, the intermediate subalgebra $M_{1} \subset$ $Q \subset M$ is not assumed a priori to be with expectation in $M$. The proof of Theorem $\mathrm{A}$ is based on a key result (see Theorem 3.1) which is a generalization of Popa's result [Po83, Lemma 2.1] regarding asymptotic orthogonality for free group factors to arbitrary free product von Neumann algebras. The proof uses Popa's original method together with $\varepsilon$-orthogonality techniques from [Ho12a, Ho12b].

In order to obtain structural results for the intermediate subalgebras $M_{1} \subset Q \subset M$, we will next assume that $Q$ is with expectation in $M$ in the statement of Corollary B. Recall that a factor $M$ (with separable predual) is full if its asymptotic centralizer $M_{\omega}$ is trivial (see [Co74]). Observe that by [AH12, Theorem 5.3], this is equivalent to $M^{\prime} \cap M^{\omega}=\mathbf{C}$.

Corollary B. Let $\left(M_{1}, \varphi_{1}\right)$ and $\left(M_{2}, \varphi_{2}\right)$ be von Neumann algebras with separable predual endowed with faithful normal states. Assume that the centralizer $M_{1}^{\varphi_{1}}$ is diffuse. Denote by $(M, \varphi)=\left(M_{1}, \varphi_{1}\right) *\left(M_{2}, \varphi_{2}\right)$ the free product von Neumann algebra.

Then any intermediate von Neumann subalgebra $M_{1} \subset Q \subset M$ with faithful normal conditional expectation $E_{Q}: M \rightarrow Q$ is globally invariant under the modular automorphism group $\left(\sigma_{t}^{\varphi}\right)$. Moreover, there exists a sequence of pairwise orthogonal projections $z_{n} \in Q^{\prime} \cap M \subset \mathcal{Z}\left(M_{1}\right)$ such that $\sum_{n} z_{n}=1$ and

- $M_{1} z_{0}=Q z_{0}$ and

- $Q z_{n}$ is a full nonamenable factor such that $\left(Q z_{n}\right)^{\prime} \cap\left(z_{n} M z_{n}\right)^{\omega}=\mathbf{C} z_{n}$ for every $n \geq 1$.

Corollary B generalizes and strengthens [Po83, Lemma 3.1] and Ge95, Lemma 4.4]. Corollary B moreover implies that if $M_{1}$ has property Gamma, then $M_{1} \subset M$ is a maximal Gamma subalgebra with expectation in $M$. The structural result in Corollary B allows us to obtain a wide range of maximal amenable subalgebras inside nonamenable factors. In particular, Corollary C below provides the first class of concrete maximal amenable subalgebras with expectation in the framework of type III factors. 
Corollary C. Any diffuse amenable von Neumann algebra with separable predual can be concretely realized as a maximal amenable subalgebra with expectation inside a full nonamenable type $\mathrm{III}_{1}$ factor.

Our main last result deals with Gamma stability for subalgebras of tracial free product von Neumann algebras. Theorem $\mathrm{D}$ below is a further generalization of Corollary $\mathrm{B}$ where the subalgebra $Q \subset M$ is only assumed to have a diffuse intersection with $M_{1}$.

Theorem D. Let $\left(M_{1}, \tau_{1}\right)$ and $\left(M_{2}, \tau_{2}\right)$ be von Neumann algebras with separable predual endowed with faithful normal tracial states. Assume that $M_{1}$ is diffuse. Denote by $(M, \tau)=$ $\left(M_{1}, \tau_{1}\right) *\left(M_{2}, \tau_{2}\right)$ the tracial free product von Neumann algebra.

Then for every von Neumann subalgebra $Q \subset M$ such that $Q \cap M_{1}$ is diffuse, there exists a central projection $z \in \mathcal{Z}\left(Q^{\prime} \cap M\right) \cap \mathcal{Z}\left(Q^{\prime} \cap M^{\omega}\right) \subset M_{1}$ such that

- $Q z \subset z M_{1} z$ and

- $\left(Q^{\prime} \cap M^{\omega}\right)(1-z)=\left(Q^{\prime} \cap M\right)(1-z)$ is discrete.

Theorem $\mathrm{D}$ shows in particular that whenever $Q \subset M$ is a subalgebra such that both $Q \cap M_{1}$ and $Q^{\prime} \cap M^{\omega}$ are diffuse, then $Q \subset M_{1}$ (see Theorem 4.1). This is a strengthening of the Gamma stability result in Theorem $\mathrm{A}$. Besides the asymptotic orthogonality property obtained in Theorem 3.1, the proof of Theorem $\mathrm{D}$ uses two more ingredients of $\mathrm{II}_{1}$ factors: Popa's intertwining techniques [Po01, Po03] and Peterson's L ${ }^{2}$-rigidity results for tracial free product von Neumann algebras [Pe06].

In Section 2, we recall a few preliminaires on free product and ultraproduct von Neumann algebras. In Section 3, we prove the key result regarding asymptotic orthogonality inside free products of arbitrary von Neumann algebras. Finally, we prove in Section 4 the main results of the paper.

Acknowledgments. I am grateful to Rémi Boutonnet and Sven Raum for their valuable comments regarding a first draft of this manuscript. I especially thank Rémi for pointing out a gap in the initial proof of Proposition 2.5. Finally, I thank the referee for carefully reading the paper and useful remarks.

\section{Preliminaries}

We fix once and for all a non-principal ultrafilter $\omega \in \beta(\mathbf{N}) \backslash \mathbf{N}$. All the von Neumann algebras that we consider in this paper are assumed to be $\sigma$-finite, that is, countably decomposable. We say that $M$ is a tracial von Neumann algebra if $M$ admits a faithful normal tracial state $\tau$.

Background on $\sigma$-finite von Neumann algebras. Let $M$ be any $\sigma$-finite von Neumann algebra. We denote by $\operatorname{Ball}(M)$ the unit ball of $M$ with respect to the uniform norm $\|\cdot\|_{\infty}$, $\mathcal{U}(M)$ the group of unitaries in $M$ and $\mathcal{Z}(M)$ the center of $M$. Let $\varphi \in M_{*}$ be a faithful normal state. We denote by $\mathrm{L}^{2}(M, \varphi)$ (or simply $\mathrm{L}^{2}(M)$ when no confusion is possible) the GNS $L^{2}$-completion of $M$ with respect to the inner product defined by $\langle x, y\rangle_{\varphi}=\varphi\left(y^{*} x\right)$ for all $x, y \in M$. We denote by $\Lambda_{\varphi}: M \rightarrow \mathrm{L}^{2}(M): x \mapsto \Lambda_{\varphi}(x)$ the canonical embedding and by $J_{\varphi}: \mathrm{L}^{2}(M) \rightarrow \mathrm{L}^{2}(M)$ the canonical conjugation. We have $x \Lambda_{\varphi}(y)=\Lambda_{\varphi}(x y)$ for all $x, y \in M$.

We say that two elements $x, y \in M$ are $\varphi$-orthogonal in $M$ if $\varphi\left(y^{*} x\right)=0$ or equivalently if the vectors $\Lambda_{\varphi}(x)$ and $\Lambda_{\varphi}(y)$ are orthogonal in the Hilbert space $\mathrm{L}^{2}(M)$. For all $x \in M$, write $\|x\|_{\varphi}=\varphi\left(x^{*} x\right)^{1 / 2}$ and $\|x\|_{\varphi}^{\sharp}=\varphi\left(x^{*} x+x x^{*}\right)^{1 / 2}$. Recall that the strong (resp. *-strong) topology on uniformly bounded subsets of $M$ coincides with the topology defined by $\|\cdot\|_{\varphi}$ (resp. $\|\cdot\|_{\varphi}^{\sharp}$ ). 
An element $x \in M$ is said to be analytic with respect to the modular automorphism group $\left(\sigma_{t}^{\varphi}\right)$ if the function $\mathbf{R} \rightarrow M: t \mapsto \sigma_{t}^{\varphi}(x)$ can be extended to an $M$-valued entire analytic function over $\mathbf{C}$.

We will be using the following standard facts.

Proposition 2.1. Let $(M, \varphi)$ be any $\sigma$-finite von Neumann algebra endowed with a faithful normal state.

(1) The subset $\mathcal{A} \subset M$ of all the elements in $M$ which are analytic with respect to the modular automorphism group $\left(\sigma_{t}^{\varphi}\right)$ forms a unital $\sigma$-strongly dense $*$-subalgebra of $M$.

(2) For all $a \in \mathcal{A}$ and all $x \in M$, we have

$$
\Lambda_{\varphi}(x a)=J_{\varphi} \sigma_{-\mathrm{i} / 2}^{\varphi}\left(a^{*}\right) J_{\varphi} \Lambda_{\varphi}(x) .
$$

(3) For all $a \in \mathcal{A}$ and all $x \in M$, we have

$$
\varphi(a x)=\varphi\left(x \sigma_{-\mathrm{i}}^{\varphi}(a)\right) .
$$

In particular, for all $a \in \mathcal{A}$ and all $x, y \in M$, we have that $x a$ and $y$ are $\varphi$-orthogonal in $M$ if and only if $x$ and $y \sigma_{\mathrm{i}}^{\varphi}(a)^{*}$ are $\varphi$-orthogonal in $M$.

Proof. (1) follows from [Ta03, Lemma VIII.2.3] and (2) follows from [Ta03, Lemma VIII.3.10]. Let us prove (3). For every $a \in \mathcal{A}$ and every $x \in M$, we have

$$
\begin{aligned}
\varphi\left(x \sigma_{-\mathrm{i}}^{\varphi}(a)\right) & =\left\langle\Lambda_{\varphi}\left(x \sigma_{-\mathrm{i}}^{\varphi}(a)\right), \Lambda_{\varphi}(1)\right\rangle_{\varphi} \\
& =\left\langle J_{\varphi} \sigma_{\mathrm{i} / 2}^{\varphi}\left(a^{*}\right) J_{\varphi} \Lambda_{\varphi}(x), \Lambda_{\varphi}(1)\right\rangle_{\varphi} \\
& =\left\langle\Lambda_{\varphi}(x), J_{\varphi} \sigma_{-\mathrm{i} / 2}^{\varphi}(a) J_{\varphi} \Lambda_{\varphi}(1)\right\rangle_{\varphi} \\
& =\left\langle\Lambda_{\varphi}(x), \Lambda_{\varphi}\left(a^{*}\right)\right\rangle_{\varphi} \\
& =\varphi(a x) .
\end{aligned}
$$

In particular, for all $a \in \mathcal{A}$ and all $x, y \in M$, we have

$$
\varphi\left(\left(y \sigma_{\mathrm{i}}^{\varphi}(a)^{*}\right)^{*} x\right)=\varphi\left(\sigma_{\mathrm{i}}^{\varphi}(a) y^{*} x\right)=\varphi\left(y^{*} x a\right) .
$$

Hence $x a$ and $y$ are $\varphi$-orthogonal in $M$ if and only if $x$ and $y \sigma_{\mathrm{i}}^{\varphi}(a)^{*}$ are $\varphi$-orthogonal in $M$.

Proposition 2.2. Let $M$ be any $\sigma$-finite von Neumann algebra.

(1) We have that $M$ is diffuse if and only if there exists a faithful normal state $\varphi \in M_{*}$ such that the centralizer $M^{\varphi}$ is diffuse. Moreover in that case, there exists a unitary $u \in \mathcal{U}\left(M^{\varphi}\right)$ such that $u^{k} \rightarrow 0$ weakly as $|k| \rightarrow \infty$.

(2) Let $N \subset M$ be a von Neumann subalgebra with expectation. If $N$ is diffuse, so is $M$.

Proof. (1) Assume first that $M$ is diffuse. There exists a sequence of pairwise orthogonal projections $z_{n} \in \mathcal{Z}(M)$ such that $\sum_{n} z_{n}=1, M z_{0}$ is a von Neumann algebra with a diffuse center and $M z_{n}$ is a diffuse factor for every $n \geq 1$. Choose any faithful normal state $\varphi_{0}$ on $M z_{0}$. By [HS90, Theorem 11.1], for every $n \geq 1$, choose a faithful normal state $\varphi_{n}$ on $M z_{n}$ such that the centralizer $\left(M z_{n}\right)^{\varphi_{n}}$ is diffuse. Let $\left(a_{n}\right)_{n}$ be a sequence of positive reals so that $\sum_{n} a_{n}=1$. The formula $\varphi=\sum_{n} a_{n} \varphi_{n}$ defines a faithful normal state on $M$ such that

$$
M^{\varphi}=\bigoplus_{n}\left(M z_{n}\right)^{\varphi_{n}} \text {. }
$$

Therefore, $M^{\varphi}$ is diffuse.

Assume next that $M^{\varphi}$ is diffuse for some faithful normal state $\varphi \in M_{*}$. Using the above decomposition, for every $n \geq 1$ such that $z_{n} \neq 0$, letting $\varphi_{n}=\frac{1}{\varphi\left(z_{n}\right)} \varphi\left(\cdot z_{n}\right)$, we have that $\left(M z_{n}\right)^{\varphi_{n}}=M^{\varphi} z_{n}$ is diffuse. Therefore $M z_{n}$ is a non-type I factor and so is diffuse. Thus, $M$ is diffuse. 
When $M^{\varphi}$ is diffuse, take $A \subset M^{\varphi}$ a maximal abelian subalgebra. Then $A$ is necessarily diffuse. Then choose a diffuse subalgebra $B \subset A$ with separable predual. Since $B \cong \mathrm{L}^{\infty}(\mathbf{T})$, we can then take a unitary $u \in \mathcal{U}(B)$ such that $u^{k} \rightarrow 0$ weakly as $|k| \rightarrow \infty$.

(2) Denote by $E: M \rightarrow N$ a faithful normal conditional expectation and choose a faithful normal state $\psi \in N_{*}$ such that $N^{\psi}$ is diffuse. Then $\varphi=\psi \circ E$ is a faithful normal state on $M$ such that $N^{\psi} \subset M^{\varphi}$. Since $N^{\psi}$ is diffuse and $M^{\varphi}$ is tracial, $M^{\varphi}$ is diffuse and so is $M$ by item (1) of the proposition.

Free product von Neumann algebras. For $i=1,2$, let $\left(M_{i}, \varphi_{i}\right)$ be any $\sigma$-finite von Neumann algebra endowed with a faithful normal state. The free product von Neumann algebra $(M, \varphi)=\left(M_{1}, \varphi_{1}\right) *\left(M_{2}, \varphi_{2}\right)$ is the von Neumann algebra $M$ generated by $M_{1}$ and $M_{2}$ where the faithful normal state $\varphi$ satisfies the following freeness condition:

$$
\varphi\left(x_{1} \cdots x_{n}\right)=0 \text { whenever } x_{j} \in M_{i_{j}} \ominus \mathbf{C} \text { and } i_{1} \neq \cdots \neq i_{n} .
$$

Here and in what follows, we denote by $M_{i} \ominus \mathbf{C}=\operatorname{ker}\left(\varphi_{i}\right)$. We refer to the product $x_{1} \cdots x_{n}$ where $x_{j} \in M_{i_{j}} \ominus \mathbf{C}$ and $i_{1} \neq \cdots \neq i_{n}$ as a reduced word in $\left(M_{i_{1}} \ominus \mathbf{C}\right) \cdots\left(M_{i_{n}} \ominus \mathbf{C}\right)$ of length $n \geq 1$. The linear span of 1 and of all the reduced words in $\left(M_{i_{1}} \ominus \mathbf{C}\right) \cdots\left(M_{i_{n}} \ominus \mathbf{C}\right)$ where $n \geq 1$ and $i_{1} \neq \cdots \neq i_{n}$ forms a unital $\sigma$-strongly dense $*$-subalgebra of $M$.

For all $n \geq 1$ and all $i_{1} \neq \cdots \neq i_{n}$, the mapping

$$
\begin{aligned}
\mathrm{L}^{2}\left(\left(M_{i_{1}} \ominus \mathbf{C}\right) \cdots(\right. & \left.\left(M_{i_{n}} \ominus \mathbf{C}\right), \varphi\right) \\
\Lambda_{\varphi}\left(x_{1} \cdots x_{n}\right) & \mapsto \Lambda_{\varphi_{i_{1}}}\left(x_{1}\left(M_{i_{1}} \ominus \mathbf{C}, \varphi_{i_{1}}\right) \otimes \cdots \otimes \Lambda_{\varphi_{i_{n}}}\left(x_{n}\right)\right.
\end{aligned}
$$

defines a unitary operator. Moreover, we have

$$
\mathrm{L}^{2}(M, \varphi)=\mathbf{C} \oplus \bigoplus_{n \geq 1} \bigoplus_{i_{1} \neq \cdots \neq i_{n}} \mathrm{~L}^{2}\left(M_{i_{1}} \ominus \mathbf{C}, \varphi_{i_{1}}\right) \otimes \cdots \otimes \mathrm{L}^{2}\left(M_{i_{n}} \ominus \mathbf{C}, \varphi_{i_{n}}\right)
$$

For all $t \in \mathbf{R}$, we have $\sigma_{t}^{\varphi}=\sigma_{t}^{\varphi_{1}} * \sigma_{t}^{\varphi_{2}}$ (see [Ba93, Lemma 1] and [Dy92, Theorem 1]). By [Ta03, Theorem IX.4.2], there exists a unique $\varphi$-preserving faithful normal conditional expectation $E_{M_{1}}: M \rightarrow M_{1}$. Moreover, we have $E_{M_{1}}\left(x_{1} \cdots x_{n}\right)=0$ for all the reduced words $x_{1} \cdots x_{n}$ which contains at least one letter from $M_{2} \ominus \mathbf{C}$ (see [Ue11, Lemma 2.1]). For more on free product von Neumann algebras, we refer the reader to [Ue98, Ue11, Vo85, Vo92].

Ultraproduct von Neumann algebras. Let $M$ be any $\sigma$-finite von Neumann algebra. Define

$$
\begin{aligned}
\mathcal{I}^{\omega}(M) & =\left\{\left(x_{n}\right)_{n} \in \ell^{\infty}(\mathbf{N}, M): x_{n} \rightarrow 0 * \text {-strongly as } n \rightarrow \omega\right\} \\
\mathcal{M}^{\omega}(M) & =\left\{\left(x_{n}\right)_{n} \in \ell^{\infty}(\mathbf{N}, M):\left(x_{n}\right)_{n} \mathcal{I}^{\omega}(M) \subset \mathcal{I}^{\omega}(M) \text { and } \mathcal{I}^{\omega}(M)\left(x_{n}\right)_{n} \subset \mathcal{I}^{\omega}(M)\right\} .
\end{aligned}
$$

We have that the multiplier algebra $\mathcal{M}^{\omega}(M)$ is a $\mathrm{C}^{*}$-algebra and $\mathcal{I}^{\omega}(M) \subset \mathcal{M}^{\omega}(M)$ is a norm closed two-sided ideal. Following [Oc85], we define the ultraproduct von Neumann algebra $M^{\omega}$ by $M^{\omega}=\mathcal{M}^{\omega}(M) / \mathcal{I}^{\omega}(M)$. We denote the image of $\left(x_{n}\right)_{n} \in \mathcal{M}^{\omega}(M)$ by $\left(x_{n}\right)^{\omega} \in M^{\omega}$.

For all $x \in M$, the constant sequence $(x)_{n}$ lies in the multiplier algebra $\mathcal{M}^{\omega}(M)$. We will then identify $M$ with $\left(M+\mathcal{I}^{\omega}(M)\right) / \mathcal{I}^{\omega}(M)$ and regard $M \subset M^{\omega}$ as a von Neumann subalgebra. The map $E_{M}: M^{\omega} \rightarrow M:\left(x_{n}\right)^{\omega} \mapsto \sigma$-weak $\lim _{n \rightarrow \omega} x_{n}$ is a faithful normal conditional expectation. For every faithful normal state $\varphi \in M_{*}$, the formula $\varphi^{\omega}=\varphi \circ E_{M}$ defines a faithful normal state on $M^{\omega}$. Observe that $\varphi^{\omega}\left(\left(x_{n}\right)^{\omega}\right)=\lim _{n \rightarrow \omega} \varphi\left(x_{n}\right)$ for all $\left(x_{n}\right)^{\omega} \in M^{\omega}$.

Let $Q \subset M$ be any von Neumann subalgebra with faithful normal conditional expectation $E_{Q}: M \rightarrow Q$. Choose a faithful normal state $\varphi$ on $Q$ and still denote by $\varphi$ the faithful normal state $\varphi \circ E_{Q}$ on $M$. We have $\ell^{\infty}(\mathbf{N}, Q) \subset \ell^{\infty}(\mathbf{N}, M), \mathcal{I}^{\omega}(Q) \subset \mathcal{I}^{\omega}(M)$ and $\mathcal{M}^{\omega}(Q) \subset$ $\mathcal{M}^{\omega}(M)$. We will then identify $Q^{\omega}=\mathcal{M}^{\omega}(Q) / \mathcal{I}^{\omega}(Q)$ with $\left(\mathcal{M}^{\omega}(Q)+\mathcal{I}^{\omega}(M)\right) / \mathcal{I}^{\omega}(M)$ and regard $Q^{\omega} \subset M^{\omega}$ as a von Neumann subalgebra. Observe that the norm $\|\cdot\|_{(\varphi \mid Q)^{\omega}}$ on $Q^{\omega}$ is 
the restriction of the norm $\|\cdot\|_{\varphi^{\omega}}$ to $Q^{\omega}$. Observe moreover that $\left(E_{Q}\left(x_{n}\right)\right)_{n} \in \mathcal{I}^{\omega}(Q)$ for all $\left(x_{n}\right)_{n} \in \mathcal{I}^{\omega}(M)$ and $\left(E_{Q}\left(x_{n}\right)\right)_{n} \in \mathcal{M}^{\omega}(Q)$ for all $\left(x_{n}\right)_{n} \in \mathcal{M}^{\omega}(M)$. Therefore, the mapping $E_{Q^{\omega}}: M^{\omega} \rightarrow Q^{\omega}:\left(x_{n}\right)^{\omega} \mapsto\left(E_{Q}\left(x_{n}\right)\right)^{\omega}$ is a well-defined conditional expectation satisfying $\varphi^{\omega} \circ E_{Q^{\omega}}=\varphi^{\omega}$. Hence, $E_{Q^{\omega}}: M^{\omega} \rightarrow Q^{\omega}$ is a faithful normal conditional expectation.

Put $\mathcal{H}=\mathrm{L}^{2}(M, \varphi)$. The ultraproduct Hilbert space $\mathcal{H}^{\omega}$ is defined to be the quotient of $\ell^{\infty}(\mathbf{N}, \mathcal{H})$ by the subspace consisting in sequences $\left(\xi_{n}\right)_{n}$ satisfying $\lim _{n \rightarrow \omega}\left\|\xi_{n}\right\|_{\mathcal{H}}=0$. We denote the image of $\left(\xi_{n}\right)_{n} \in \ell^{\infty}(\mathbf{N}, \mathcal{H})$ by $\left(\xi_{n}\right)_{\omega} \in \mathcal{H}^{\omega}$. The inner product space structure on the Hilbert space $\mathcal{H}^{\omega}$ is defined by $\left\langle\left(\xi_{n}\right)_{\omega},\left(\eta_{n}\right)_{\omega}\right\rangle_{\mathcal{H}^{\omega}}=\lim _{n \rightarrow \omega}\left\langle\xi_{n}, \eta_{n}\right\rangle_{\mathcal{H}}$. The GNS Hilbert space $\mathrm{L}^{2}\left(M^{\omega}, \varphi^{\omega}\right)$ can be embedded into $\mathcal{H}^{\omega}$ as a closed subspace by $\Lambda_{\varphi^{\omega}}\left(\left(x_{n}\right)^{\omega}\right) \mapsto\left(\Lambda_{\varphi}\left(x_{n}\right)\right)_{\omega}$. For more on ultraproduct von Neumann algebras, we refer the reader to [AH12, Oc85].

Put $x \varphi=\varphi(\cdot x)$ and $\varphi x=\varphi(x \cdot)$ for all $x \in M$ and all $\varphi \in M_{*}$. We will be using the following standard facts.

Lemma 2.3. Let $(M, \varphi)$ be any $\sigma$-finite von Neumann algebra endowed with a faithful normal state. Then for every $x \in M$, we have

$$
\|x \varphi\| \leq\|x\|_{\varphi},\|\varphi x\| \leq\left\|x^{*}\right\|_{\varphi} \text { and }\|x \varphi-\varphi x\|=\left\|x^{*} \varphi-\varphi x^{*}\right\| .
$$

Proof. Let $x \in M$. Using the Cauchy-Schwarz inequality, for all $y \in \operatorname{Ball}(M)$, we have

$$
|(x \varphi)(y)|=|\varphi(y x)| \leq\left\|y^{*}\right\|_{\varphi}\|x\|_{\varphi} \leq\|x\|_{\varphi}
$$

and hence $\|x \varphi\| \leq\|x\|_{\varphi}$. Likewise, for all $y \in \operatorname{Ball}(M)$, we have

$$
|(\varphi x)(y)|=|\varphi(x y)| \leq\left\|x^{*}\right\|_{\varphi}\|y\|_{\varphi} \leq\left\|x^{*}\right\|_{\varphi}
$$

and hence $\|\varphi x\| \leq\left\|x^{*}\right\|_{\varphi}$. Moreover, for all $y \in \operatorname{Ball}(M)$, we have

$$
\left|\left(x^{*} \varphi-\varphi x^{*}\right)(y)\right|=\left|\varphi\left(y x^{*}-x^{*} y\right)\right|=\left|\overline{\varphi\left(y x^{*}-x^{*} y\right)}\right|=\left|\varphi\left(x y^{*}-y^{*} x\right)\right|=\left|(x \varphi-\varphi x)\left(y^{*}\right)\right| .
$$

This implies that $\|x \varphi-\varphi x\|=\left\|x^{*} \varphi-\varphi x^{*}\right\|$.

Proposition 2.4. Let $(M, \varphi)$ be any $\sigma$-finite von Neumann algebra endowed with a faithful normal state.

(1) For every $\left(x_{n}\right)_{n} \in \mathcal{M}^{\omega}(M)$ and every $\left(y_{n}\right)_{n} \in \ell^{\infty}(\mathbf{N}, M)$ such that $x_{n}-y_{n} \rightarrow 0 *$ strongly as $n \rightarrow \omega$, we have $\left(y_{n}\right)_{n} \in \mathcal{M}^{\omega}(M)$ and $\left(x_{n}\right)^{\omega}=\left(y_{n}\right)^{\omega} \in M^{\omega}$.

(2) For every $\left(x_{n}\right)_{n} \in \ell^{\infty}(\mathbf{N}, M)$ satisfying $\lim _{n \rightarrow \omega}\left\|x_{n} \varphi-\varphi x_{n}\right\|=0$, we have $\left(x_{n}\right)_{n} \in$ $\mathcal{M}^{\omega}(M)$ and $\left(x_{n}\right)^{\omega} \in\left(M^{\omega}\right)^{\varphi^{\omega}}$.

(3) For every projection $e \in M^{\omega}$, there exists a sequence of projections $\left(e_{n}\right)_{n} \in \mathcal{M}^{\omega}(M)$ such that $e=\left(e_{n}\right)^{\omega}$.

Proof. (1) Let $\left(x_{n}\right)_{n} \in \mathcal{M}^{\omega}(M)$ and $\left(y_{n}\right)_{n} \in \ell^{\infty}(\mathbf{N}, M)$ such that $x_{n}-y_{n} \rightarrow 0 *$-strongly as $n \rightarrow \omega$. Then $\left(y_{n}-x_{n}\right)_{n} \in \mathcal{I}^{\omega}(M) \subset \mathcal{M}^{\omega}(M)$ and hence $\left(y_{n}\right)_{n}=\left(y_{n}-x_{n}\right)_{n}+\left(x_{n}\right)_{n} \in \mathcal{M}^{\omega}(M)$. Moreover, by the definition of the ultraproduct von Neumann algebra $M^{\omega}$, we have $\left(x_{n}\right)^{\omega}=$ $\left(y_{n}\right)^{\omega} \in M^{\omega}$.

(2) Let $\left(x_{n}\right)_{n} \in \ell^{\infty}(\mathbf{N}, M)$ such that $\lim _{n \rightarrow \omega}\left\|x_{n} \varphi-\varphi x_{n}\right\|=0$. Let $\left(b_{n}\right)_{n} \in \mathcal{I}^{\omega}(M)$. We may assume that $\max \left\{\left\|x_{n}\right\|_{\infty},\left\|b_{n}\right\|_{\infty}: n \in \mathbf{N}\right\} \leq 1$. Using the Cauchy-Schwarz inequality, for all $n \in \mathbf{N}$, we have

$$
\begin{aligned}
\left(\left\|x_{n} b_{n}\right\|_{\varphi}^{\sharp}\right)^{2} & =\varphi\left(b_{n}^{*} x_{n}^{*} x_{n} b_{n}\right)+\varphi\left(x_{n} b_{n} b_{n}^{*} x_{n}^{*}\right) \\
& \leq\left\|b_{n}\right\|_{\varphi}\left\|x_{n}^{*} x_{n} b_{n}\right\|_{\varphi}+\left|\left(x_{n} \varphi-\varphi x_{n}\right)\left(b_{n} b_{n}^{*} x_{n}^{*}\right)\right|+\left|\varphi\left(b_{n} b_{n}^{*} x_{n}^{*} x_{n}\right)\right| \\
& \leq\left\|b_{n}\right\|_{\varphi}+\left\|x_{n} \varphi-\varphi x_{n}\right\|\left\|b_{n} b_{n}^{*} x_{n}^{*}\right\|_{\infty}+\left\|b_{n}^{*}\right\|_{\varphi}\left\|b_{n}^{*} x_{n}^{*} x_{n}\right\|_{\varphi} \\
& \leq\left\|b_{n}\right\|_{\varphi}+\left\|x_{n} \varphi-\varphi x_{n}\right\|+\left\|b_{n}^{*}\right\|_{\varphi} .
\end{aligned}
$$


Therefore, we obtain $\lim _{n \rightarrow \omega}\left\|x_{n} b_{n}\right\|_{\varphi}^{\sharp}=0$ and so $\left(x_{n} b_{n}\right)_{n} \in \mathcal{I}^{\omega}(M)$. Likewise, for all $n \in \mathbf{N}$, we have

$$
\begin{aligned}
\left(\left\|b_{n} x_{n}\right\|_{\varphi}^{\sharp}\right)^{2} & =\varphi\left(x_{n}^{*} b_{n}^{*} b_{n} x_{n}\right)+\varphi\left(b_{n} x_{n} x_{n}^{*} b_{n}^{*}\right) \\
& \leq\left|\left(x_{n}^{*} \varphi-\varphi x_{n}^{*}\right)\left(b_{n}^{*} b_{n} x_{n}\right)\right|+\left|\varphi\left(b_{n}^{*} b_{n} x_{n} x_{n}^{*}\right)\right|+\left\|b_{n}^{*}\right\|_{\varphi}\left\|x_{n} x_{n}^{*} b_{n}^{*}\right\|_{\varphi} \\
& \leq\left\|x_{n}^{*} \varphi-\varphi x_{n}^{*}\right\|\left\|b_{n}^{*} b_{n} x_{n}\right\|_{\infty}+\left\|b_{n}\right\|_{\varphi}\left\|b_{n} x_{n} x_{n}^{*}\right\|_{\varphi}+\left\|b_{n}^{*}\right\|_{\varphi} \\
& \leq\left\|x_{n} \varphi-\varphi x_{n}\right\|+\left\|b_{n}\right\|_{\varphi}+\left\|b_{n}^{*}\right\|_{\varphi} .
\end{aligned}
$$

Therefore, we obtain $\lim _{n \rightarrow \omega}\left\|b_{n} x_{n}\right\|_{\varphi}^{\sharp}=0$ and so $\left(b_{n} x_{n}\right)_{n} \in \mathcal{I}^{\omega}(M)$. This shows that $\left(x_{n}\right)_{n} \in$ $\mathcal{M}^{\omega}(M)$. Moreover, $x=\left(x_{n}\right)^{\omega} \in\left(M^{\omega}\right)^{\varphi^{\omega}}$ by [AH12, Lemma 4.35].

(3) The proof is identical to the one of [Co75a, Proposition 1.1.3]. Let $e \in M^{\omega}$ be any projection. We may choose a sequence $\left(x_{n}\right)_{n} \in \mathcal{M}^{\omega}(M)$ such that $\left\|x_{n}\right\|_{\infty} \leq 1$ for all $n \in \mathbf{N}$ and $e=\left(x_{n}\right)^{\omega}$. Put $y_{n}=x_{n}^{*} x_{n}$ for all $n \in \mathbf{N}$. Since $e=e^{*} e$, we have $\lim _{n \rightarrow \omega}\left\|x_{n}-y_{n}\right\|_{\varphi}^{\sharp}=0,\left(y_{n}\right)_{n} \in \mathcal{M}^{\omega}(M)$ and $e=\left(y_{n}\right)^{\omega}$. Since $e=e^{2}$, we moreover have $\lim _{n \rightarrow \omega}\left\|y_{n}-y_{n}^{2}\right\|_{\varphi}^{\sharp}=0$. Put $\varepsilon_{n}=\left\|y_{n}-y_{n}^{2}\right\|_{\varphi}$. Letting $e_{n}=\mathbf{1}_{\left[1-\sqrt{\varepsilon_{n}}, 1\right]}\left(y_{n}\right) \in M$ for all $n \in \mathbf{N}$, we have $\lim _{n \rightarrow \omega}\left\|y_{n}-e_{n}\right\|_{\varphi}^{\sharp}=0$ by [Co75a, Lemma 1.1.5]. It follows that $\left(e_{n}\right)_{n} \in \mathcal{M}^{\omega}(M)$ and $e=\left(e_{n}\right)^{\omega} \in M^{\omega}$ by item (1) of the proposition.

The next proposition will be useful to prove Corollary $\mathrm{B}$,

Proposition 2.5. Let $M$ be any factor with separable predual and $Q \subset M$ any irreducible subfactor with expectation. Then, either $Q^{\prime} \cap M^{\omega}=\mathbf{C}$ or $Q^{\prime} \cap M^{\omega}$ is diffuse.

Proof. Denote by $E_{Q}: M \rightarrow Q$ the faithful normal conditional expectation. Choose a faithful normal state on $Q$ and still denote by $\varphi$ the faithful normal state $\varphi \circ E_{Q}$ on $M$. Since $Q$ is globally invariant under the modular automorphism group $\left(\sigma_{t}^{\varphi}\right)$ and since $\sigma_{t}^{\varphi^{\omega}}(x)=\sigma_{t}^{\varphi}(x)$ for all $x \in M$, the relative commutant $Q^{\prime} \cap M^{\omega}$ is globally invariant under the modular automorphism group $\left(\sigma_{t}^{\varphi^{\omega}}\right)$. Hence $\left(Q^{\prime} \cap M^{\omega}\right)^{\varphi^{\omega}}=\left(Q^{\prime} \cap M^{\omega}\right) \cap\left(M^{\omega}\right)^{\varphi^{\omega}}=Q^{\prime} \cap\left(M^{\omega}\right)^{\varphi^{\omega}}$.

Claim. Either $Q^{\prime} \cap\left(M^{\omega}\right)^{\varphi^{\omega}}=\mathbf{C}$ or $Q^{\prime} \cap\left(M^{\omega}\right)^{\varphi^{\omega}}$ is diffuse.

Proof of the Claim. We use the proof of [Io12, Lemma 2.7]. Put $\mathcal{Q}=Q^{\prime} \cap\left(M^{\omega}\right)^{\varphi^{\omega}}$ and denote by $e \in \mathcal{Z}(\mathcal{Q})$ the maximum central projection in $\mathcal{Q}$ such that $\mathcal{Q} e$ is discrete. We may represent $e=\left(e_{n}\right)^{\omega}$ by a sequence of projections $\left(e_{n}\right)_{n} \in \mathcal{M}^{\omega}(M)$. Put $\lambda=\varphi^{\omega}(e)=\lim _{n \rightarrow \omega} \varphi\left(e_{n}\right)$. Since $Q^{\prime} \cap M=\mathbf{C}$, we have $e_{n} \rightarrow \lambda 1 \sigma$-weakly as $n \rightarrow \omega$.

Next, we construct by induction a sequence of projections $\left(f_{m}\right)_{m \geq 1}$ in $\mathcal{Q}$ such that

$$
\varphi^{\omega}\left(e f_{i}\right)=\lambda^{2} \text { and } \varphi^{\omega}\left(e f_{i} f_{j}\right)=\lambda^{3}, \forall 1 \leq i<j .
$$

Indeed, assume that $f_{1}, \ldots, f_{m} \in \mathcal{Q}$ have been constructed. For every $1 \leq j \leq m$, represent $f_{j}=\left(f_{j, n}\right)^{\omega}$ by a sequence of projections $\left(f_{j, n}\right)_{n} \in \mathcal{M}^{\omega}(M)$. Let $\left(x_{i}\right)_{i \in \mathbf{N}}$ be a $\|\cdot\|_{\varphi}^{\sharp}$-dense sequence in $\operatorname{Ball}(Q)$. Since $e=\left(e_{n}\right)^{\omega} \in\left(M^{\omega}\right)^{\varphi^{\omega}}$, since $\lim _{n \rightarrow \omega}\left\|e_{n} x_{i}-x_{i} e_{n}\right\|_{\varphi}^{\sharp}=0$ for all $i \in \mathbf{N}$ and since $e_{n} \rightarrow \lambda 1 \sigma$-weakly as $n \rightarrow \omega$, we can find an increasing sequence $\left(k_{n}\right)_{n}$ in $\mathbf{N}$ such that for every $n \geq 1$, we have

(P1) $\left\|e_{k_{n}} \varphi-\varphi e_{k_{n}}\right\| \leq \frac{1}{n}$

(P2) $\left\|e_{k_{n}} x_{i}-x_{i} e_{k_{n}}\right\|_{\varphi}^{\sharp} \leq \frac{1}{n}$ for all $1 \leq i \leq n$,

(P3) $\left|\varphi\left(e_{n} e_{k_{n}}\right)-\lambda \varphi\left(e_{n}\right)\right| \leq \frac{1}{n}$ and

(P4) $\left|\varphi\left(e_{n} f_{j, n} e_{k_{n}}\right)-\lambda \varphi\left(e_{n} f_{j, n}\right)\right| \leq \frac{1}{n}$ for all $1 \leq j \leq m$.

Property (P1) together with Proposition 2.4 imply that the sequence $\left(e_{k_{n}}\right)_{n}$ lies in the multiplier algebra $\mathcal{M}^{\omega}(M)$ and $f=\left(e_{k_{n}}\right)^{\omega} \in\left(M^{\omega}\right)^{\varphi^{\omega}}$. Property (P2) implies that $x_{i} f=f x_{i}$ for all $i \in \mathbf{N}$. Since $\left\{x_{i}: i \in \mathbf{N}\right\}$ is $*$-strongly dense in $\operatorname{Ball}(Q)$, we obtain that $f \in Q^{\prime} \cap\left(M^{\omega}\right)^{\varphi^{\omega}}=\mathcal{Q}$. 
Finally, Property (P3) implies that $\varphi^{\omega}(e f)=\lambda \varphi^{\omega}(e)=\lambda^{2}$ and Property (P4) together with the induction hypothesis imply that $\varphi^{\omega}\left(e f_{j} f\right)=\lambda \varphi^{\omega}\left(e f_{j}\right)=\lambda^{3}$ for all $1 \leq j \leq m$. We can now put $f_{m+1}=f$. This finishes the proof of the induction.

Define $p_{m}=f_{m} e$ which is a projection in $\mathcal{Q} e$. Observe that since $\mathcal{Q} e$ is a discrete tracial von Neumann algebra, $\mathcal{Q} e$ is $*$-isomorphic to a countable direct sum of finite dimensional factors and hence its unit ball $\operatorname{Ball}(\mathcal{Q} e)$ is $\|\cdot\|_{\varphi_{e}^{\omega}}$-compact, where $\varphi_{e}^{\omega}=\frac{\varphi^{\omega}(e \cdot e)}{\varphi^{\omega}(e)}$. Thus, we may choose a

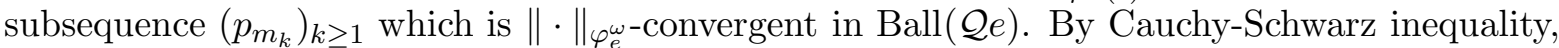
for all $1 \leq j<k$, we have

$$
\left|\varphi_{e}^{\omega}\left(p_{m_{j}} p_{m_{k}}\right)-\varphi_{e}^{\omega}\left(p_{m_{j}}\right)\right|=\left|\varphi_{e}^{\omega}\left(p_{m_{j}}\left(p_{m_{k}}-p_{m_{j}}\right)\right)\right| \leq\left\|p_{m_{j}}-p_{m_{k}}\right\|_{\varphi_{e}^{\omega}} .
$$

Taking the limit as $(j, k) \rightarrow \infty$ and using (1), we obtain $\lambda^{2}=\lambda^{3}$. Therefore $\lambda \in\{0,1\}$ and so $e \in\{0,1\}$.

This implies that either $e=0$ and $\mathcal{Q}$ is diffuse or $e=1$ and $\mathcal{Q}$ is a discrete tracial von Neumann algebra. In the case when $\mathcal{Q}$ is a discrete tracial von Neumann algebra, we show that $\mathcal{Q}=\mathbf{C}$. Assume by contradiction that $\mathcal{Q}$ is a discrete tracial von Neumann algebra and that $\mathcal{Q} \neq \mathbf{C}$.

Denote by $E_{M}: M^{\omega} \rightarrow M$ the canonical faithful normal conditional expectation. Recall that $\varphi \circ E_{M}=\varphi^{\omega}$. Since $\mathcal{Q} \neq \mathbf{C}$, we may choose a projection $e \in \mathcal{Q}$ satisfying $\varphi^{\omega}(e)=\lambda$ with $\lambda \neq 0,1$. We may represent $e=\left(e_{n}\right)^{\omega} \in \mathcal{Q}$ by a sequence of projections $\left(e_{n}\right)_{n} \in \mathcal{M}^{\omega}(M)$. Observe that $E_{M}(e)=\lambda 1=\sigma$-weak $\lim _{n \rightarrow \omega} e_{n}$. Then for all $y \in \operatorname{Ball}(M)$, we have

$$
\|e-y\|_{\varphi^{\omega}} \geq\left\|e-E_{M}(e)\right\|_{\varphi^{\omega}}=\sqrt{\lambda-\lambda^{2}}>0 .
$$

Put $\varepsilon=\frac{\sqrt{\lambda-\lambda^{2}}}{2}$. Put $e_{1}=e \in \mathcal{Q}$. Next, we construct by induction a sequence of projections $e_{m} \in \mathcal{Q}$ such that $\left\|e_{p}-e_{q}\right\|_{\varphi^{\omega}} \geq \varepsilon$ for all $p, q \geq 1$ such that $p \neq q$. Assume that $e_{1}, \ldots, e_{m} \in \mathcal{Q}$ have been constructed. For every $1 \leq j \leq m$, represent $e_{j}=\left(e_{j, n}\right)^{\omega}$ by a sequence of projections $\left(e_{j, n}\right)_{n} \in \mathcal{M}^{\omega}(M)$. Let $\left(x_{i}\right)_{i \in \mathbf{N}}$ be a $\|\cdot\|_{\varphi}^{\sharp}$-dense sequence in Ball $(Q)$. Since $e=\left(e_{n}\right)^{\omega} \in\left(M^{\omega}\right)^{\varphi^{\omega}}$, since $\lim _{k \rightarrow \omega}\left\|e_{k} x_{i}-x_{i} e_{k}\right\|_{\varphi}^{\sharp}=0$ for all $i \in \mathbf{N}$ and since $\lim _{k \rightarrow \omega}\left\|e_{k}-e_{j, n}\right\|_{\varphi}=\left\|e-e_{j, n}\right\|_{\varphi^{\omega}} \geq 2 \varepsilon$ for all $1 \leq j \leq m$ and all $n \in \mathbf{N}$, we can find an increasing sequence $\left(k_{n}\right)_{n}$ in $\mathbf{N}$ such that for every $n \geq 1$, we have

(P1) $\left\|e_{k_{n}} \varphi-\varphi e_{k_{n}}\right\| \leq \frac{1}{n}$

(P2) $\left\|e_{k_{n}} x_{i}-x_{i} e_{k_{n}}\right\|_{\varphi}^{\sharp} \leq \frac{1}{n}$ for all $1 \leq i \leq n$ and

(P3) $\left\|e_{k_{n}}-e_{j, n}\right\|_{\varphi} \geq \varepsilon$ for all $1 \leq j \leq m$.

By the same reasoning as before, Properties (P1) and (P2) imply that $\left(e_{k_{n}}\right)_{n} \in \mathcal{M}^{\omega}(M)$ and $f=\left(e_{k_{n}}\right)^{\omega} \in \mathcal{Q}$. Moreover, Property (P3) implies that $\left\|f-e_{j}\right\|_{\varphi^{\omega}} \geq \varepsilon$ for all $1 \leq j \leq m$. We can now put $e_{m+1}=f$. This finishes the proof of the induction.

So, we have constructed a sequence of projections $e_{m} \in \mathcal{Q}$ such that $\left\|e_{p}-e_{q}\right\|_{\varphi^{\omega}} \geq \varepsilon$ for all $p, q \geq 1$ such that $p \neq q$. This however contradicts the fact that $\operatorname{Ball}(\mathcal{Q})$ is $\|\cdot\|_{\varphi^{\omega} \text {-compact }}$ and finishes the proof of the Claim.

Assume that $Q^{\prime} \cap\left(M^{\omega}\right)^{\varphi^{\omega}}=\mathbf{C}$. Then by AH12, Lemma 5.4], we have that $Q^{\prime} \cap M^{\omega}=\mathbf{C}$ or $Q^{\prime} \cap M^{\omega}$ is a type III $_{1}$ factor. Next, assume that $Q^{\prime} \cap\left(M^{\omega}\right)^{\varphi^{\omega}}$ is diffuse. Then, using Proposition 2.2, we have that $Q^{\prime} \cap M^{\omega}$ is diffuse. Therefore, either $Q^{\prime} \cap M^{\omega}=\mathbf{C}$ or $Q^{\prime} \cap M^{\omega}$ is diffuse.

Proposition 2.6. For every diffuse amenable von Neumann algebra $M$ with separable predual, the central sequence algebra $M^{\prime} \cap M^{\omega}$ is diffuse.

Proof. Let $M$ be any diffuse amenable von Neumann algebra with separable predual. There exists a sequence of pairwise orthogonal projections $z_{n} \in \mathcal{Z}(M)$ such that $\sum_{n} z_{n}=1, M z_{0}$ is an amenable von Neumann algebra with a diffuse center and separable predual and $M z_{n}$ is a diffuse 
amenable factor with separable predual for every $n \geq 1$. It is obvious that $\left(M z_{0}\right)^{\prime} \cap\left(M z_{0}\right)^{\omega}$ is diffuse. By the classification of amenable factors with separable predual (see [Co72, Co74, Co75b, Co85, Ha84]), $M z_{n}$ is hyperfinite and $\left(M z_{n}\right)^{\prime} \cap\left(M z_{n}\right)^{\omega}$ is diffuse for every $n \geq 1$. Therefore $M^{\prime} \cap M^{\omega}=\bigoplus_{n}\left(M z_{n}\right)^{\prime} \cap\left(M z_{n}\right)^{\omega}$ is diffuse.

An elementary fact on $\varepsilon$-orthogonality. Let $\mathcal{H}$ be a complex Hilbert space and $\varepsilon \geq 0$. We say that two (not necessarily closed) subspaces $\mathcal{K}, \mathcal{L} \subset \mathcal{H}$ are $\varepsilon$-orthogonal and we denote by $\mathcal{K} \perp_{\varepsilon} \mathcal{L}$ if

$$
\left|\langle\xi, \eta\rangle_{\mathcal{H}}\right| \leq \varepsilon\|\xi\|_{\mathcal{H}}\|\eta\|_{\mathcal{H}}, \forall \xi \in \mathcal{K}, \forall \eta \in \mathcal{L}
$$

Define the function

$$
\delta:\left[0, \frac{1}{2}\right) \rightarrow \mathbf{R}_{+}: t \mapsto \frac{2 t}{\sqrt{1-t-\sqrt{2} t \sqrt{1-t}}} .
$$

We will be using the following elementary fact regarding $\varepsilon$-orthogonality whose proof can be found in [Ho12a, Proposition 2.3].

Proposition 2.7 ([Ho12a $)$. Let $k \geq 1$. Let $0 \leq \varepsilon<1$ such that $\delta^{\circ(k-1)}(\varepsilon)<1$. For all $1 \leq i \leq 2^{k}$, let $p_{i} \in \mathbf{B}(\mathcal{H})$ be projections such that $p_{i} \mathcal{H} \perp_{\varepsilon} p_{j} \mathcal{H}$ for all $i, j \in\left\{1, \ldots, 2^{k}\right\}$ such that $i \neq j$. Write $P_{k}=\bigvee_{i=1}^{2^{k}} p_{i}$. Then for all $\xi \in \mathcal{H}$, we have

$$
\sum_{i=1}^{2^{k}}\left\|p_{i} \xi\right\|_{\mathcal{H}}^{2} \leq \prod_{j=0}^{k-1}\left(1+\delta^{\circ j}(\varepsilon)\right)^{2}\left\|P_{k} \xi\right\|_{\mathcal{H}}^{2} .
$$

\section{AsYmptotic ORTHOGONALity In THE ULTRAPRODUCT FRAMEWORK}

The key result of the paper is the following generalization of Popa's result [Po83, Lemma 2.1] regarding asymptotic orthogonality for free group factors to arbitrary free product von Neumann algebras. There are mainly two difficulties that arise in generalizing Popa's result [Po83, Lemma 2.1] to the setting of arbitrary free product von Neumann algebras. The first main difficulty is that the free product von Neumann algebra $(M, \varphi)=\left(M_{1}, \varphi_{1}\right) *\left(M_{2}, \varphi_{2}\right)$ is no longer assumed to be tracial. Hence, we need to work in the ultraproduct von Neumann algebra framework and carefully approximate elements in $M$ in the $\sigma$-strong topology by finite linear combinations of reduced words which are analytic with respect to the modular automorphism group $\left(\sigma_{t}^{\varphi}\right)$ (see also the proof of [Ue11, Proposition 3.5] where a similar method is used). The second main difficulty is that unlike the case of the free group factors, $M$ is no longer assumed to have a nice basis of unitary elements. To circumvent this issue, we will use $\varepsilon$-orthogonality techniques from [Ho12a, Ho12b].

Theorem 3.1. Let $\left(M_{1}, \varphi_{1}\right)$ and $\left(M_{2}, \varphi_{2}\right)$ be $\sigma$-finite von Neumann algebras endowed with faithful normal states. Assume that the centralizer $M_{1}^{\varphi_{1}}$ is diffuse. Denote by $(M, \varphi)=$ $\left(M_{1}, \varphi_{1}\right) *\left(M_{2}, \varphi_{2}\right)$ the free product von Neumann algebra.

Let $u \in \mathcal{U}\left(M_{1}^{\varphi_{1}}\right)$ be any unitary such that $u^{k} \rightarrow 0$ weakly as $|k| \rightarrow \infty$. For every $x \in\{u\}^{\prime} \cap M^{\omega}$ and every $y \in M \ominus M_{1}$, the elements $y\left(x-E_{M_{1}^{\omega}}(x)\right),\left(x-E_{M_{1}^{\omega}}(x)\right) y$ and $y E_{M_{1}^{\omega}}(x)-E_{M_{1}^{\omega}}(x) y$ are pairwise $\varphi^{\omega}$-orthogonal in $M^{\omega}$.

Proof. For every $i \in\{1,2\}$, denote by $\mathcal{A}_{i} \subset M_{i}$ (resp. $\mathcal{A} \subset M$ ) the unital $\sigma$-strongly dense *-subalgebra of all the elements in $M_{i}$ (resp. $M$ ) which are analytic with respect to the modular automorphism group $\left(\sigma_{t}^{\varphi_{i}}\right)$ (resp. $\left(\sigma_{t}^{\varphi}\right)$ ) (see Proposition 2.11). Observe that for every $i \in\{1,2\}$, $\mathcal{A}_{i} \subset \mathcal{A}$. Denote by $\left(\mathcal{A}_{i_{1}} \ominus \mathbf{C}\right) \cdots\left(\mathcal{A}_{i_{n}} \ominus \mathbf{C}\right)$ the set of all the reduced words of the form $a_{1} \cdots a_{n}$ with $a_{j} \in \mathcal{A}_{i_{j}} \ominus \mathbf{C}, n \geq 1$ and $i_{1} \neq \cdots \neq i_{n}$. The linear span of

$$
\left\{1,\left(\mathcal{A}_{i_{1}} \ominus \mathbf{C}\right) \cdots\left(\mathcal{A}_{i_{n}} \ominus \mathbf{C}\right): n \geq 1, i_{1} \neq \cdots \neq i_{n}\right\}
$$

forms a unital $\sigma$-strongly dense $*$-subalgebra of $M$. 
Using the existence of the normal conditional expectation $E_{M_{1}}: M \rightarrow M_{1}$, every $y \in M \ominus M_{1}$ can be approximated with respect to the $\sigma$-strong topology by a net $\left(y_{\alpha}\right)_{\alpha \in I}$ of finite linear combinations of reduced words in $\left(\mathcal{A}_{i_{1}} \ominus \mathbf{C}\right) \cdots\left(\mathcal{A}_{i_{n}} \ominus \mathbf{C}\right)$ where $n \geq 1,2 \in\left\{i_{1}, \ldots, i_{n}\right\}$ and $i_{1} \neq \cdots \neq i_{n}$. Assume that for every $\alpha \in I$ and every $x \in\{u\}^{\prime} \cap M^{\omega}, y_{\alpha}\left(x-E_{M_{1}^{\omega}}(x)\right)$, $\left(x-E_{M_{1}^{\omega}}(x)\right) y_{\alpha}$ and $y_{\alpha} E_{M_{1}^{\omega}}(x)-E_{M_{1}^{\omega}}(x) y_{\alpha}$ are pairwise $\varphi^{\omega}$-orthogonal in $M^{\omega}$. Then since $y_{\alpha} \rightarrow y \sigma$-strongly as $\alpha \rightarrow \infty$, it follows that

$$
\begin{aligned}
y_{\alpha}\left(x-E_{M_{1}^{\omega}}(x)\right) & \rightarrow y\left(x-E_{M_{1}^{\omega}}(x)\right) \\
\left(x-E_{M_{1}^{\omega}}(x)\right) y_{\alpha} & \rightarrow\left(x-E_{M_{1}^{\omega}}(x)\right) y \\
y_{\alpha} E_{M_{1}^{\omega}}(x)-E_{M_{1}^{\omega}}(x) y_{\alpha} & \rightarrow y E_{M_{1}^{\omega}}(x)-E_{M_{1}^{\omega}}(x) y
\end{aligned}
$$

$\sigma$-strongly as $\alpha \rightarrow \infty$. Therefore, $y\left(x-E_{M_{1}^{\omega}}(x)\right),\left(x-E_{M_{1}^{\omega}}(x)\right) y$ and $y E_{M_{1}^{\omega}}(x)-E_{M_{1}^{\omega}}(x) y$ are pairwise $\varphi^{\omega}$-orthogonal in $M^{\omega}$. Using the previous discussion, we infer that it suffices to prove the result for

$$
y=\sum_{j=1}^{k} w_{j} \text { where } w_{j}=a_{j, 1} b_{j, 1} \cdots b_{j, n_{j}} a_{j, n_{j}+1}
$$

with $n_{j} \geq 1, a_{j, 1}=1$ or $a_{j, 1} \in \mathcal{A}_{1} \ominus \mathbf{C}, a_{j, n_{j}+1}=1$ or $a_{j, n_{j}+1} \in \mathcal{A}_{1} \ominus \mathbf{C}, a_{j, 2}, \ldots, a_{j, n_{j}} \in \mathcal{A}_{1} \ominus \mathbf{C}$ and $b_{j, 1}, \ldots, b_{j, n_{j}} \in \mathcal{A}_{2} \ominus \mathbf{C}$. We fix such an element $y \in M \ominus M_{1}$ until the end of the proof. Observe that for every $1 \leq j \leq k$, we have $w_{j} \in \mathcal{A} \ominus \mathbf{C}$ and

$$
\sigma_{-\mathrm{i}}^{\varphi}\left(w_{j}^{*}\right)=\sigma_{-\mathrm{i}}^{\varphi_{1}}\left(a_{j, n_{j}+1}^{*}\right) \sigma_{-\mathrm{i}}^{\varphi_{2}}\left(b_{j, n_{j}}^{*}\right) \cdots \sigma_{-\mathrm{i}}^{\varphi_{2}}\left(b_{j, 1}^{*}\right) \sigma_{-\mathrm{i}}^{\varphi_{1}}\left(a_{j, 1}^{*}\right) \text {. }
$$

It follows that $\sigma_{-\mathrm{i}}^{\varphi}\left(w_{j}^{*}\right)$ is a reduced word containing at least one letter from $M_{2} \ominus \mathbf{C}$.

Denote by $V \subset M_{1}$ the finite dimensional vector subspace generated by 1 and by

- the first letters coming from $M_{1} \ominus \mathbf{C}$ of the reduced words $w_{i}, w_{i}^{*}, \sigma_{-\mathrm{i}}^{\varphi}\left(w_{i}^{*}\right)$ and the first letters coming from $M_{1} \ominus \mathbf{C}$ of all the reduced words arising in the finite linear decomposition of $w_{j}^{*} w_{i}$ into reduced words, for all $1 \leq i, j \leq k$, and

- the last letters coming from $M_{1} \ominus \mathbf{C}$ of the reduced words $w_{i}$ and the last letters coming from $M_{1} \ominus \mathbf{C}$ of all the reduced words arising in the finite linear decomposition of $w_{i} \sigma_{-\mathrm{i}}^{\varphi}\left(w_{j}^{*}\right)$ into reduced words, for all $1 \leq i, j \leq k$.

Let $\ell=\operatorname{dim}(V)$ and choose elements $e_{1}, \ldots, e_{\ell} \in V$ so that $\left(\Lambda_{\varphi_{1}}\left(e_{i}\right)\right)_{i=1}^{\ell}$ forms an orthonormal basis for $\Lambda_{\varphi_{1}}(V)$. By Gram-Schmidt process, choose a vector subspace $W \subset M_{1}$ so that

$$
\mathrm{L}^{2}\left(M_{1}\right)=\Lambda_{\varphi_{1}}(V) \oplus \overline{\Lambda_{\varphi_{1}}(W)} .
$$

We will be using the following notation:

- $\mathcal{K}_{1} \subset \mathrm{L}^{2}(M)$ is the closed subspace generated by the image under $\Lambda_{\varphi}$ of the linear span of all the reduced words in $\left(M_{2} \ominus \mathbf{C}\right) \cdots\left(M_{2} \ominus \mathbf{C}\right),(V \ominus \mathbf{C})\left(M_{2} \ominus \mathbf{C}\right) \cdots\left(M_{2} \ominus \mathbf{C}\right)$, $\left(M_{2} \ominus \mathbf{C}\right) \cdots\left(M_{2} \ominus \mathbf{C}\right)\left(M_{1} \ominus \mathbf{C}\right)$ and $(V \ominus \mathbf{C})\left(M_{2} \ominus \mathbf{C}\right) \cdots\left(M_{2} \ominus \mathbf{C}\right)\left(M_{1} \ominus \mathbf{C}\right)$. Observe that

$$
\mathcal{K}_{1} \cong \Lambda_{\varphi}(V) \otimes \mathrm{L}^{2}\left(\left(M_{2} \ominus \mathbf{C}\right) \cdots\left(M_{2} \ominus \mathbf{C}\right) M_{1}\right) .
$$

- $\mathcal{K}_{2} \subset \mathrm{L}^{2}(M)$ is the closed subspace generated by the image under $\Lambda_{\varphi}$ of the linear span of all the reduced words in $W\left(M_{2} \ominus \mathbf{C}\right) \cdots\left(M_{2} \ominus \mathbf{C}\right)$ and $W\left(M_{2} \ominus \mathbf{C}\right) \cdots\left(M_{2} \ominus \mathbf{C}\right)(V \ominus \mathbf{C})$. Observe that

$$
\mathcal{K}_{2} \cong \mathrm{L}^{2}\left(W\left(M_{2} \ominus \mathbf{C}\right) \cdots\left(M_{2} \ominus \mathbf{C}\right)\right) \otimes \Lambda_{\varphi}(V) .
$$

- $\mathcal{L} \subset \mathrm{L}^{2}(M)$ is the closed subspace generated by the image under $\Lambda_{\varphi}$ of the linear span of all the reduced words in $W\left(M_{2} \ominus \mathbf{C}\right) \cdots\left(M_{2} \ominus \mathbf{C}\right) W$. Observe that

$$
\mathrm{L}^{2}\left(M_{1}\right) \oplus \mathcal{K}_{1} \oplus \mathcal{K}_{2} \oplus \mathcal{L}=\mathrm{L}^{2}(M) .
$$


Let $u \in \mathcal{U}\left(M_{1}^{\varphi_{1}}\right)$ such that $u^{k} \rightarrow 0$ weakly as $|k| \rightarrow \infty$ and put $T=u J_{\varphi} u J_{\varphi} \in \mathcal{U}\left(\mathrm{L}^{2}(M)\right)$. Observe that since $u \in \mathcal{U}\left(M_{1}^{\varphi_{1}}\right) \subset \mathcal{U}\left(M^{\varphi}\right)$, we have $T \Lambda_{\varphi}(z)=\Lambda_{\varphi}\left(u z u^{*}\right)$ for all $z \in M$.

Claim 1. For all $\varepsilon>0$, there exists $k_{0} \in \mathbf{N}$ such that for all $i \in\{1,2\}$ and all $|k| \geq k_{0}$, we have $T^{k} \mathcal{K}_{i} \perp_{\varepsilon} \mathcal{K}_{i}$.

Proof of Claim 1. Let $\xi, \eta \in \mathcal{K}_{1}$ that we write $\sum_{i=1}^{\ell} \Lambda_{\varphi}\left(e_{i}\right) \otimes \xi_{i}$ and $\eta=\sum_{j=1}^{\ell} \Lambda_{\varphi}\left(e_{j}\right) \otimes \eta_{j}$ with $\xi_{i}, \eta_{j} \in \mathrm{L}^{2}\left(\left(M_{2} \ominus \mathbf{C}\right) \cdots\left(M_{2} \ominus \mathbf{C}\right) M_{1}\right)$. Observe that $\|\xi\|_{\varphi}^{2}=\sum_{i=1}^{\ell}\left\|\xi_{i}\right\|_{\varphi}^{2}$ and $\|\eta\|_{\varphi}^{2}=$ $\sum_{j=1}^{\ell}\left\|\eta_{j}\right\|_{\varphi}^{2}$. Since $u \in M^{\varphi}$, we have $T^{k} \xi=\sum_{i=1}^{\ell} \Lambda_{\varphi}\left(u^{k} e_{i}\right) \otimes J_{\varphi} u^{k} J_{\varphi} \xi_{i}$ and hence

$$
\left|\left\langle T^{k} \xi, \eta\right\rangle_{\varphi}\right| \leq \sum_{i, j=1}^{\ell}\left|\varphi\left(e_{j}^{*} u^{k} e_{i}\right)\right|\left\|\xi_{i}\right\|_{\varphi}\left\|\eta_{j}\right\|_{\varphi} .
$$

Since $u^{k} \rightarrow 0$ weakly as $|k| \rightarrow \infty$, we may choose $k_{1} \in \mathbf{N}$ such that for all $|k| \geq k_{1}$ and all $1 \leq i, j \leq \ell$, we have $\left|\varphi\left(e_{j}^{*} u^{k} e_{i}\right)\right| \leq \varepsilon / \ell$. By Cauchy-Schwarz inequality, for all $|k| \geq k_{1}$, we obtain $\left|\left\langle T^{k} \xi, \eta\right\rangle_{\varphi}\right| \leq \varepsilon\|\xi\|_{\varphi}\|\eta\|_{\varphi}$.

Likewise let $\xi, \eta \in \mathcal{K}_{2}$ that we write $\sum_{i=1}^{\ell} \xi_{i} \otimes \Lambda_{\varphi}\left(e_{i}\right)$ and $\eta=\sum_{j=1}^{\ell} \eta_{j} \otimes \Lambda_{\varphi}\left(e_{j}\right)$ with $\xi_{i}, \eta_{j} \in$ $\mathrm{L}^{2}\left(W\left(M_{2} \ominus \mathbf{C}\right) \cdots\left(M_{2} \ominus \mathbf{C}\right)\right)$. Observe that $\|\xi\|_{\varphi}^{2}=\sum_{i=1}^{\ell}\left\|\xi_{i}\right\|_{\varphi}^{2}$ and $\|\eta\|_{\varphi}^{2}=\sum_{j=1}^{\ell}\left\|\eta_{j}\right\|_{\varphi}^{2}$. Since $u \in M^{\varphi}$, we have $T^{k} \xi=\sum_{i=1}^{\ell} u^{k} \xi_{i} \otimes \Lambda_{\varphi}\left(e_{i} u^{-k}\right)$ and hence

$$
\left|\left\langle T^{k} \xi, \eta\right\rangle_{\varphi}\right| \leq \sum_{i, j=1}^{\ell}\left|\varphi\left(e_{j}^{*} e_{i} u^{-k}\right)\right|\left\|\xi_{i}\right\|_{\varphi}\left\|\eta_{j}\right\|_{\varphi} .
$$

Since $u^{k} \rightarrow 0$ weakly as $|k| \rightarrow \infty$, we may choose $k_{2} \in \mathbf{N}$ such that for all $|k| \geq k_{2}$ and all $1 \leq i, j \leq \ell$, we have $\left|\varphi\left(e_{j}^{*} e_{i} u^{-k}\right)\right| \leq \varepsilon / \ell$. By Cauchy-Schwarz inequality, for all $|k| \geq k_{2}$, we obtain $\left|\left\langle T^{k} \xi, \eta\right\rangle_{\varphi}\right| \leq \varepsilon\|\xi\|_{\varphi}\|\eta\|_{\varphi}$.

Put $k_{0}=\max \left(k_{1}, k_{2}\right)$. Then for all $i \in\{1,2\}$ and all $|k| \geq k_{0}$, we have that $T^{k} \mathcal{K}_{i} \perp_{\varepsilon} \mathcal{K}_{i}$.

Claim 2. For all $i \in\{1,2\}$ and all $\left(z_{n}\right)^{\omega} \in\{u\}^{\prime} \cap M^{\omega}$, we have

$$
\lim _{n \rightarrow \omega}\left\|P_{\mathcal{K}_{i}}\left(\Lambda_{\varphi}\left(z_{n}\right)\right)\right\|_{\varphi}=0 \text {. }
$$

Proof of Claim Q Let $i \in\{1,2\}$ and $z=\left(z_{n}\right)^{\omega} \in\{u\}^{\prime} \cap M^{\omega}$. We may assume that $\left\|z_{n}\right\|_{\infty} \leq 1$ for all $n \in \mathbf{N}$. For all $n \in \mathbf{N}$ and all $k \in \mathbf{N}$, we have

$$
\begin{aligned}
\left\|P_{\mathcal{K}_{i}}\left(\Lambda_{\varphi}\left(z_{n}\right)\right)\right\|_{\varphi}^{2} & =\left\|T^{k} P_{\mathcal{K}_{i}}\left(\Lambda_{\varphi}\left(z_{n}\right)\right)\right\|_{\varphi}^{2} \\
& =\left\|T^{k} P_{\mathcal{K}_{i}}\left(\Lambda_{\varphi}\left(z_{n}\right)\right)-P_{T^{k} \mathcal{K}_{i}}\left(\Lambda_{\varphi}\left(z_{n}\right)\right)+P_{T^{k}} \mathcal{K}_{i}\left(\Lambda_{\varphi}\left(z_{n}\right)\right)\right\|_{\varphi}^{2} \\
& \leq 2\left\|T^{k} P_{\mathcal{K}_{i}}\left(\Lambda_{\varphi}\left(z_{n}\right)\right)-P_{T^{k} \mathcal{K}_{i}}\left(\Lambda_{\varphi}\left(z_{n}\right)\right)\right\|_{\varphi}^{2}+2\left\|P_{T^{k} \mathcal{K}_{i}}\left(\Lambda_{\varphi}\left(z_{n}\right)\right)\right\|_{\varphi}^{2} \\
& =2\left\|P_{T^{k} \mathcal{K}_{i}}\left(\Lambda_{\varphi}\left(u^{k} z_{n} u^{-k}-z_{n}\right)\right)\right\|_{\varphi}^{2}+2\left\|P_{T^{k}} \mathcal{K}_{i}\left(\Lambda_{\varphi}\left(z_{n}\right)\right)\right\|_{\varphi}^{2} \\
& \leq 2\left\|u^{k} z_{n} u^{-k}-z_{n}\right\|_{\varphi}^{2}+2\left\|P_{T^{k} \mathcal{K}_{i}}\left(\Lambda_{\varphi}\left(z_{n}\right)\right)\right\|_{\varphi}^{2} .
\end{aligned}
$$

Fix $K \geq 1$. Choose $\varepsilon>0$ very small according to [Ho12a, Proposition 2.3] so that $\prod_{j=0}^{K-1}(1+$ $\left.\delta^{\circ j}(\varepsilon)\right)^{2} \leq 2$. Then choose a subset $\mathcal{G} \subset \mathbf{N}$ of $2^{K}$ integers such that two distinct integers in $\mathcal{G}$ are at least at distance $k_{0}$ from one another. By Claim 1, we obtain $T^{k_{1}} \mathcal{K}_{i} \perp_{\varepsilon} T^{k_{2}} \mathcal{K}_{i}$ for all $k_{1}, k_{2} \in \mathcal{G}$ such that $k_{1} \neq k_{2}$. Thus, we obtain

$$
2^{K}\left\|P_{\mathcal{K}_{i}}\left(\Lambda_{\varphi}\left(z_{n}\right)\right)\right\|_{\varphi}^{2} \leq 2 \sum_{k \in \mathcal{G}}\left\|u^{k} z_{n} u^{-k}-z_{n}\right\|_{\varphi}^{2}+4\left\|z_{n}\right\|_{\varphi}^{2} .
$$

Since $\mathcal{G}$ is finite, we have $\lim _{n \rightarrow \omega}\left\|P_{\mathcal{K}_{i}}\left(\Lambda_{\varphi}\left(z_{n}\right)\right)\right\|_{\varphi}^{2} \leq 2^{2-K}$ for all $K \geq 1$. Therefore, we obtain $\lim _{n \rightarrow \omega}\left\|P_{\mathcal{K}_{i}}\left(\Lambda_{\varphi}\left(z_{n}\right)\right)\right\|_{\varphi}=0$. 
Claim 3. The subspaces $y \mathcal{L}, J_{\varphi} \sigma_{-\mathrm{i} / 2}^{\varphi}\left(y^{*}\right) J_{\varphi} \mathcal{L}$ and $y \mathrm{~L}^{2}\left(M_{1}\right)+J_{\varphi} \sigma_{-\mathrm{i} / 2}^{\varphi}\left(y^{*}\right) J_{\varphi} \mathrm{L}^{2}\left(M_{1}\right)$ are pairwise orthogonal in $\mathrm{L}^{2}(M)$.

Proof of Claim 3. Recall that $y=\sum_{j=1}^{k} w_{j}$ where $w_{j}=a_{j, 1} b_{j, 1} \cdots b_{j, n_{j}} a_{j, n_{j}+1}$ with $n_{j} \geq 1$, $a_{j, 1}=1$ or $a_{j, 1} \in \mathcal{A}_{1} \ominus \mathbf{C}, a_{j, n_{j}+1}=1$ or $a_{j, n_{j}+1} \in \mathcal{A}_{1} \ominus \mathbf{C}, a_{j, 2}, \ldots, a_{j, n_{j}} \in \mathcal{A}_{1} \ominus \mathbf{C}$ and $b_{j, 1}, \ldots, b_{j, n_{j}} \in \mathcal{A}_{2} \ominus \mathbf{C}$. Observe that

$$
\begin{aligned}
y \mathcal{L} & \subset \overline{\operatorname{span}\left\{\Lambda_{\varphi}\left(w_{j} W\left(M_{2} \ominus \mathbf{C}\right) \cdots\left(M_{2} \ominus \mathbf{C}\right) W\right): 1 \leq j \leq k\right\}} \\
J_{\varphi} \sigma_{-\mathrm{i} / 2}^{\varphi}\left(y^{*}\right) J_{\varphi} \mathcal{L} & \subset \overline{\operatorname{span}\left\{\Lambda_{\varphi}\left(W\left(M_{2} \ominus \mathbf{C}\right) \cdots\left(M_{2} \ominus \mathbf{C}\right) W w_{j}\right): 1 \leq j \leq k\right\}}
\end{aligned}
$$

and

$$
y \mathrm{~L}^{2}\left(M_{1}\right)+J_{\varphi} \sigma_{-\mathrm{i} / 2}^{\varphi}\left(y^{*}\right) J_{\varphi} \mathrm{L}^{2}\left(M_{1}\right) \subset \overline{\operatorname{span}\left\{\Lambda_{\varphi}\left(w_{i} M_{1}\right), \Lambda_{\varphi}\left(M_{1} w_{j}\right): 1 \leq i, j \leq k\right\}} .
$$

Let $1 \leq i \leq k$. Observe that by the choice of the vector subspace $W \subset M_{1}$, any letter $v \in W$ is $\varphi$-orthogonal in $M$ to the first letter of the reduced word $w_{i}^{*}$ and to the first letter of the reduced word $\sigma_{-\mathrm{i}}^{\varphi}\left(w_{i}^{*}\right)$. Hence $w_{i} v$ is a reduced word starting with the first letter of $w_{i}$ and ending with a letter from $M_{1} \ominus \mathbf{C}$ and $v w_{i}$ is a reduced word starting with a letter from $M_{1} \ominus \mathbf{C}$ and ending with the last letter of $w_{i}$. Moreover both $v w_{i}$ and $w_{i} v$ contain at least one letter from $M_{2} \ominus \mathbf{C}$.

Let $1 \leq i, j \leq k$. By the choice of the vector subspace $W \subset M_{1}$ and the remark above, the first letter of any reduced word $w_{i} v$ with $v \in W$ is $\varphi$-orthogonal to $W$ in $M$. This implies that $W\left(M_{2} \ominus \mathbf{C}\right) \cdots\left(M_{2} \ominus \mathbf{C}\right) W w_{j}$ and $w_{i} W\left(M_{2} \ominus \mathbf{C}\right) \cdots\left(M_{2} \ominus \mathbf{C}\right) W$ are $\varphi$-orthogonal in $M$. Since this holds for all $1 \leq i, j \leq k$, using (2) and (3), we obtain that the subspaces $y \mathcal{L}$ and $J_{\varphi} \sigma_{-\mathrm{i} / 2}^{\varphi}\left(y^{*}\right) J_{\varphi} \mathcal{L}$ are orthogonal in $\mathrm{L}^{2}(M)$.

Let $1 \leq i, j \leq k$. If $n_{i} \leq n_{j}$, then any element in $w_{i} M_{1}$ is a finite linear combination of reduced words which have at most $n_{i}$ letters from $M_{2} \ominus \mathbf{C}$ while a reduced word in $w_{j} W\left(M_{2} \ominus \mathbf{C}\right) \cdots\left(M_{2} \ominus\right.$ C) $W$ has at least $n_{j}+1$ letters from $M_{2} \ominus \mathbf{C}$. This implies that $w_{j} W\left(M_{2} \ominus \mathbf{C}\right) \cdots\left(M_{2} \ominus \mathbf{C}\right) W$ and $w_{i} M_{1}$ are $\varphi$-orthogonal in $M$. If $n_{i}>n_{j}$, then $w_{j}^{*} w_{i}$ is a finite linear combination of reduced words whose first letter is $\varphi$-orthogonal to $W$ in $M$ and which contain at least one letter from $M_{2} \ominus \mathbf{C}$. It follows that any element in $w_{j}^{*} w_{i} M_{1}$ is a finite linear combination of reduced words whose first letter is $\varphi$-orthogonal to $W$ in $M$. Again, this implies that $w_{j} W\left(M_{2} \ominus \mathbf{C}\right) \cdots\left(M_{2} \ominus \mathbf{C}\right) W$ and $w_{i} M_{1}$ are $\varphi$-orthogonal in $M$. Next, since $w_{i}$ contains at least one letter from $M_{2} \ominus \mathbf{C}$ and by the choice of the vector subspace $W \subset M_{1}$, any element in $M_{1} w_{i}$ is a finite linear combination of reduced words whose last letter is $\varphi$-orthogonal to $W$ in $M$. This implies that $w_{j} W\left(M_{2} \ominus \mathbf{C}\right) \cdots\left(M_{2} \ominus \mathbf{C}\right) W$ and $M_{1} w_{i}$ are $\varphi$-orthogonal in $M$. Since the previous reasoning holds for all $1 \leq i, j \leq k$, using (2) and (4), we obtain that the subspaces $y \mathcal{L}$ and $y \mathrm{~L}^{2}\left(M_{1}\right)+J_{\varphi} \sigma_{-\mathrm{i} / 2}^{\varphi}\left(y^{*}\right) J_{\varphi} \mathrm{L}^{2}\left(M_{1}\right)$ are orthogonal in $\mathrm{L}^{2}(M)$.

Let $1 \leq i, j \leq k$. Since $w_{i}$ contains at least one letter from $M_{2} \ominus \mathbf{C}$ and by the choice of the vector subspace $W \subset M_{1}$, any element in $w_{i} M_{1}$ is a finite linear combination of reduced words whose first letter is $\varphi$-orthogonal to $W$ in $M$. This implies that $W\left(M_{2} \ominus \mathbf{C}\right) \cdots\left(M_{2} \ominus \mathbf{C}\right) W w_{j}$ and $w_{i} M_{1}$ are $\varphi$-orthogonal in $M$. Next, if $n_{i} \leq n_{j}$, then any element in $M_{1} w_{i}$ is a finite linear combination of reduced words which have at most $n_{i}$ letters from $M_{2} \ominus \mathbf{C}$ while a reduced word in $W\left(M_{2} \ominus \mathbf{C}\right) \cdots\left(M_{2} \ominus \mathbf{C}\right) W w_{j}$ has at least $n_{j}+1$ letters from $M_{2} \ominus \mathbf{C}$. This implies that $W\left(M_{2} \ominus \mathbf{C}\right) \cdots\left(M_{2} \ominus \mathbf{C}\right) W w_{j}$ and $M_{1} w_{i}$ are $\varphi$-orthogonal in $M$. If $n_{i}>n_{j}$, then $w_{i} \sigma_{-\mathrm{i}}^{\varphi}\left(w_{j}^{*}\right)$ is a finite linear combination of reduced words whose last letter is $\varphi$-orthogonal to $W$ in $M$ and which contain at least one letter from $M_{2} \ominus \mathbf{C}$. It follows that any element in $M_{1} w_{i} \sigma_{-\mathrm{i}}^{\varphi}\left(w_{j}^{*}\right)$ is a finite linear combination of reduced words whose last letter is $\varphi$-orthogonal to $W$ in $M$. Using Proposition 2.1, this implies again that $W\left(M_{2} \ominus \mathbf{C}\right) \cdots\left(M_{2} \ominus \mathbf{C}\right) W w_{j}$ and $M_{1} w_{i}$ are $\varphi$-orthogonal in $M$. Since the previous reasoning holds for all $1 \leq i, j \leq k$, using (3) and 
(44), we obtain that the subspaces $J_{\varphi} \sigma_{-\mathrm{i} / 2}^{\varphi}\left(y^{*}\right) J_{\varphi} \mathcal{L}$ and $y \mathrm{~L}^{2}\left(M_{1}\right)+J_{\varphi} \sigma_{-\mathrm{i} / 2}^{\varphi}\left(y^{*}\right) J_{\varphi} \mathrm{L}^{2}\left(M_{1}\right)$ are orthogonal in $\mathrm{L}^{2}(M)$. This finishes the proof of Claim 3 ,

We are now ready to finish the proof of Theorem 3.1. Let $x \in\{u\}^{\prime} \cap M^{\omega}$ and put $z=$ $x-E_{M_{1}^{\omega}}(x)$. Observe that since $u \in M_{1} \subset M_{1}^{\omega}$, we have $z \in\{u\}^{\prime} \cap\left(M^{\omega} \ominus M_{1}^{\omega}\right)$. Write $z=\left(z_{n}\right)^{\omega} \in\{u\}^{\prime} \cap\left(M^{\omega} \ominus M_{1}^{\omega}\right)$ with $z_{n}=x_{n}-E_{M_{1}}\left(x_{n}\right)$. By Claim 2 and since $y$ is analytic with respect to the modular automorphism group $\left(\sigma_{t}^{\varphi}\right)$, we obtain

$$
\begin{aligned}
\Lambda_{\varphi^{\omega}}(y z) & =\left(\Lambda_{\varphi}\left(y z_{n}\right)\right)_{\omega}=\left(y \Lambda_{\varphi}\left(z_{n}\right)\right)_{\omega} \\
& =\left(y P_{\mathcal{L}}\left(\Lambda_{\varphi}\left(z_{n}\right)\right)\right)_{\omega} \in \mathrm{L}^{2}(M)^{\omega} \\
\Lambda_{\varphi^{\omega}}(z y) & =\left(\Lambda_{\varphi}\left(z_{n} y\right)\right)_{\omega}=\left(J_{\varphi} \sigma_{-\mathrm{i} / 2}(y)^{*} J_{\varphi} \Lambda_{\varphi}\left(z_{n}\right)\right)_{\omega} \\
& =\left(J_{\varphi} \sigma_{-\mathrm{i} / 2}^{\varphi}(y)^{*} J_{\varphi} P_{\mathcal{L}}\left(\Lambda_{\varphi}\left(z_{n}\right)\right)\right)_{\omega} \in \mathrm{L}^{2}(M)^{\omega} \\
\Lambda_{\varphi^{\omega}}\left(y E_{M_{1}^{\omega}}(x)-E_{M_{1}^{\omega}}(x) y\right) & =\left(\Lambda_{\varphi}\left(y E_{M_{1}}\left(x_{n}\right)-E_{M_{1}}\left(x_{n}\right) y\right)\right)_{\omega} \\
& =\left(\left(y-J_{\varphi} \sigma_{-\mathrm{i} / 2}^{\varphi}(y)^{*} J_{\varphi}\right) \Lambda_{\varphi}\left(E_{M_{1}}\left(x_{n}\right)\right)\right)_{\omega} \in \mathrm{L}^{2}(M)^{\omega} .
\end{aligned}
$$

Using Claim 3 for every $n \in \mathbf{N}$ and using the ultraproduct Hilbert space structure of $\mathrm{L}^{2}(M)^{\omega}$, we obtain that $\Lambda_{\varphi^{\omega}}\left(y\left(x-E_{M_{1}^{\omega}}(x)\right)\right), \Lambda_{\varphi^{\omega}}\left(\left(x-E_{M_{1}^{\omega}}(x)\right) y\right)$ and $\Lambda_{\varphi^{\omega}}\left(y E_{M_{1}^{\omega}}(x)-E_{M_{1}^{\omega}}(x) y\right)$ are pairwise orthogonal in $\mathrm{L}^{2}(M)^{\omega}$. This implies that $y\left(x-E_{M_{1}^{\omega}}(x)\right),\left(x-E_{M_{1}^{\omega}}(x)\right) y$ and $y E_{M_{1}^{\omega}}(x)-E_{M_{1}^{\omega}}(x) y$ are pairwise $\varphi^{\omega}$-orthogonal in $M^{\omega}$.

\section{Proofs of the Main Results}

\subsection{Proof of Theorem $\mathbf{A}$ and Corollaries $\mathbf{B}$ and $\mathbf{C}$,}

Proof of Theorem $\AA$, Let $M_{1} \subset Q \subset M$ be any intermediate von Neumann subalgebra such that $Q^{\prime} \cap M^{\omega}$ is diffuse. Since $M_{1}^{\varphi_{1}}$ is diffuse, by [Ue11, Corollary 3.2], we have $Q^{\prime} \cap M \subset$ $M_{1}^{\prime} \cap M \subset M_{1}$ and so $Q^{\prime} \cap M=\mathcal{Z}(Q)=Q^{\prime} \cap M_{1} \subset \mathcal{Z}\left(M_{1}\right)$.

First, denote by $z \in Q^{\prime} \cap M$ the maximum projection such that $M_{1} z=Q z$. We show that $z=1$. Assume by contradiction that $z \neq 1$ and put $q=z^{\perp}=1-z \in Q^{\prime} \cap M=\mathcal{Z}(Q)$. We have $q \neq 0$ and $Q q \ominus M_{1} q \neq 0$. Denote by $\mathcal{J}$ the nonzero $\sigma$-strongly closed two-sided ideal in $Q q$ generated by $Q q \ominus M_{1} q$. Let $e \in \mathcal{Z}(Q q)=\mathcal{Z}(Q) q$ be the unique nonzero central projection in $Q q$ such that $\mathcal{J}=Q e$. We necessarily have $e=q$. Indeed otherwise we have $q-e \neq 0$ and by the choice of the projection $z \in Q^{\prime} \cap M$, we have $Q(q-e) \ominus M_{1}(q-e) \neq 0$. Now let $y \in Q(q-e) \ominus M_{1}(q-e)$ such that $y \neq 0$. Since $y \in Q q \ominus M_{1} q$, we obtain $y \in \mathcal{J}$ and so $y=y e$. However since $y \in Q(q-e) \ominus M_{1}(q-e)$, we also obtain $y=y(q-e)$ and thus $y=0$. This is a contradiction. Thus, we have $e=q$.

Next, we show that $(Q q)^{\prime} \cap(q M q)^{\omega} \subset\left(M_{1} q\right)^{\omega}$. Indeed, let $x \in(Q q)^{\prime} \cap(q M q)^{\omega} \subset M_{1}^{\prime} \cap M^{\omega}$. For every $y \in Q q \ominus M_{1} q \subset M \ominus M_{1}$, we have

$$
0=y x-x y=y\left(x-E_{M_{1}^{\omega}}(x)\right)-\left(x-E_{M_{1}^{\omega}}(x)\right) y+\left(y E_{M_{1}^{\omega}}(x)-E_{M_{1}^{\omega}}(x) y\right) .
$$

By Theorem 3.1, $y\left(x-E_{M_{1}^{\omega}}(x)\right),\left(x-E_{M_{1}^{\omega}}(x)\right) y$ and $\left(y E_{M_{1}^{\omega}}(x)-E_{M_{1}^{\omega}}(x) y\right)$ are pairwise $\varphi^{\omega}$ orthogonal in $M^{\omega}$. By Pythagora's theorem, we obtain $y\left(x-E_{M_{1}^{\omega}}(x)\right)=0$. Likewise, for every $a \in Q q$ and every $y \in Q q \ominus M_{1} q$, we have $a y\left(x-E_{M_{1}^{\omega}}(x)\right)=0$ and since $y E_{M_{1}}(a) \in Q q \ominus M_{1} q$ and $a-E_{M_{1}}(a) \in Q q \ominus M_{1} q$, we also have

$$
\text { y } a\left(x-E_{M_{1}^{\omega}}(x)\right)=y E_{M_{1}}(a)\left(x-E_{M_{1}^{\omega}}(x)\right)+y\left(a-E_{M_{1}}(a)\right)\left(x-E_{M_{1}^{\omega}}(x)\right)=0 .
$$

This implies that for every $y \in \mathcal{J}$, we have $y\left(x-E_{M_{1}^{\omega}}(x)\right)=0$ hence $q\left(x-E_{M_{1}^{\omega}}(x)\right)=0$. Therefore, $x=E_{\left(M_{1} q\right)^{\omega}}(x) \in\left(M_{1} q\right)^{\omega}$. 
Now we have that $(Q q)^{\prime} \cap(q M q)^{\omega}=(Q q)^{\prime} \cap\left(M_{1} q\right)^{\omega}$. Since $Q^{\prime} \cap M^{\omega}$ is diffuse and since $(Q q)^{\prime} \cap(q M q)^{\omega}=q\left(Q^{\prime} \cap M^{\omega}\right) q$, we have that $(Q q)^{\prime} \cap\left(M_{1} q\right)^{\omega}$ is diffuse as well. This implies that there exists a net of unitaries $U_{\alpha} \in \mathcal{U}\left((Q q)^{\prime} \cap\left(M_{1} q\right)^{\omega}\right)$ such that $U_{\alpha} \rightarrow 0$ weakly as $\alpha \rightarrow \infty$. We may represent every $U_{\alpha} \in \mathcal{U}\left((Q q)^{\prime} \cap\left(M_{1} q\right)^{\omega}\right)$ by a sequence of elements $\left(u_{n}^{\alpha}\right)_{n} \in \mathcal{M}^{\omega}\left(M_{1} q\right)$ such that $u_{n}^{\alpha} \in \operatorname{Ball}\left(M_{1} q\right)$ for every $\alpha$ and every $n \in \mathbf{N}, U_{\alpha}=\left(u_{n}^{\alpha}\right)^{\omega}$ for every $\alpha$ and $y u_{n}^{\alpha}-u_{n}^{\alpha} y \rightarrow 0$ $*$-strongly as $n \rightarrow \omega$ for every $\alpha$ and every $y \in Q q$.

Define the directed set

$$
\mathcal{I}=\left\{i=(\varepsilon, \mathcal{F}, \mathcal{G}): \varepsilon>0, \mathcal{F} \subset M_{1} q \text { and } \mathcal{G} \subset Q q \text { are finite subsets }\right\}
$$

with order relation given by

$$
\left(\varepsilon_{1}, \mathcal{F}_{1}, \mathcal{G}_{1}\right) \leq\left(\varepsilon_{2}, \mathcal{F}_{2}, \mathcal{G}_{2}\right) \text { if and only if } \varepsilon_{2} \leq \varepsilon_{1}, \mathcal{F}_{1} \subset \mathcal{F}_{2} \text { and } \mathcal{G}_{1} \subset \mathcal{G}_{2} .
$$

Let $i=(\varepsilon, \mathcal{F}, \mathcal{G}) \in \mathcal{I}$. Since $U_{\alpha} \rightarrow 0$ weakly as $\alpha \rightarrow \infty$, there exists $\alpha$ such that $\left|\varphi^{\omega}\left(b^{*} U_{\alpha} a\right)\right| \leq$ $\varepsilon / 2$ for all $a, b \in \mathcal{F}$. Since $U_{\alpha}=\left(u_{n}^{\alpha}\right)^{\omega} \in \mathcal{U}\left((Q q)^{\prime} \cap\left(M_{1} q\right)^{\omega}\right)$, for all $a, b \in \mathcal{F}$ and all $c \in \mathcal{G}$, we have

$$
\begin{aligned}
\frac{\varepsilon}{2} \geq\left|\varphi^{\omega}\left(b^{*} U_{\alpha} a\right)\right| & =\lim _{n \rightarrow \omega}\left|\varphi\left(b^{*} u_{n}^{\alpha} a\right)\right| \\
\|a\|_{\varphi}=\left\|U_{\alpha} a\right\|_{\varphi^{\omega}} & =\lim _{n \rightarrow \omega}\left\|u_{n}^{\alpha} a\right\|_{\varphi} \\
0=\left\|c U_{\alpha}-U_{\alpha} c\right\|_{\varphi^{\omega}} & =\lim _{n \rightarrow \omega}\left\|c u_{n}^{\alpha}-u_{n}^{\alpha} c\right\|_{\varphi} .
\end{aligned}
$$

Since $\mathcal{F} \subset M_{1} q$ and $\mathcal{G} \subset Q q$ are finite subsets, there exists $n=n(\alpha)$ such that

$$
\max \left\{\left|\|a\|_{\varphi}-\left\|u_{n(\alpha)}^{\alpha} a\right\|_{\varphi}\right|,\left\|c u_{n(\alpha)}^{\alpha}-u_{n(\alpha)}^{\alpha} c\right\|_{\varphi},\left|\varphi\left(b^{*} u_{n(\alpha)}^{\alpha} a\right)\right|: a, b \in \mathcal{F}, c \in \mathcal{G}\right\} \leq \varepsilon .
$$

Put $w_{i}=u_{n(\alpha)}^{\alpha} \in \operatorname{Ball}\left(M_{1} q\right)$. Thus, $\left(w_{i}\right)_{i \in \mathcal{I}}$ is a net of elements in $\operatorname{Ball}\left(M_{1} q\right)$ such that

(P1) $\lim _{i \in \mathcal{I}}\left\|w_{i} a\right\|_{\varphi}=\|a\|_{\varphi}$ for all $a \in M_{1} q$.

(P2) $\lim _{i \in \mathcal{I}}\left\|c w_{i}-w_{i} c\right\|_{\varphi}=0$ for all $c \in Q q$.

(P3) $\lim _{i \in \mathcal{I}}\left|\varphi\left(b^{*} w_{i} a\right)\right|=0$ for all $a, b \in M_{1} q$.

Put $\mathcal{E}=\operatorname{span}\left(\left\{q\left(M_{i_{1}} \ominus \mathbf{C}\right) \cdots\left(M_{i_{n}} \ominus \mathbf{C}\right) q: n \geq 1,2 \in\left\{i_{1}, \ldots, i_{n}\right\}\right.\right.$ and $\left.\left.i_{1} \neq \cdots \neq i_{n}\right\}\right)$. Observe that $\mathcal{E}$ is $\sigma$-strongly dense in $q M q \ominus M_{1} q$.

Claim. The following hold true.

(1) For all $a, b \in \mathcal{E}$, we have

$$
\lim _{i \in \mathcal{I}}\left\|E_{M_{1} q}\left(b^{*} w_{i} a\right)\right\|_{\varphi}=0 .
$$

(2) For all $b \in \mathcal{E}$ and all $y \in q M q \ominus M_{1} q$, we have

$$
\lim _{i \in \mathcal{I}}\left\|E_{M_{1} q}\left(b^{*} w_{i} y\right)\right\|_{\varphi}=0 .
$$

Proof of the Claim. (1) By linearity, it suffices to prove the result for all the elements $a, b \in \mathcal{E}$ of the form $a=a_{1} \cdots a_{2 m+1}$ and $b=b_{1} \cdots b_{2 n+1}$ with $m, n \geq 1, a_{1}=q$ or $a_{1} \in M_{1} q \ominus \mathbf{C} q$, $a_{2 m+1}=q$ or $a_{2 m+1} \in M_{1} q \ominus \mathbf{C} q, b_{1}=q$ or $b_{1} \in M_{1} q \ominus \mathbf{C} q, b_{2 n+1}=q$ or $b_{2 n+1} \in M_{1} q \ominus \mathbf{C} q$, $a_{2}, \ldots, a_{2 m}, b_{2}, \ldots, b_{2 n} \in M_{2} \ominus \mathbf{C}$ and $a_{3}, \ldots a_{2 m-1}, b_{3}, \ldots, b_{2 n-1} \in M_{1} \ominus \mathbf{C}$. We have

$$
b^{*} w_{i} a=b_{2 n+1}^{*} \cdots b_{2}^{*}\left(b_{1}^{*} w_{i} a_{1}\right) a_{2} \cdots a_{2 m+1} .
$$

By the freeness property, we have

$$
E_{M_{1}}\left(b^{*} w_{i} a\right)=\varphi\left(b_{1}^{*} w_{i} a_{1}\right) E_{M_{1}}\left(b_{2 n+1}^{*} \cdots b_{2}^{*} a_{2} \cdots a_{2 m+1}\right) .
$$

Using property $(\mathrm{P} 3)$ of the net $\left(w_{i}\right)_{i \in \mathcal{I}}$, we obtain $\lim _{i \in \mathcal{I}}\left\|E_{M_{1} q}\left(b^{*} w_{i} a\right)\right\|_{\varphi}=0$. 
(2) Let $y \in q M q \ominus M_{1} q$ and $b \in \mathcal{E}$. We may assume that $\|b\|_{\infty} \leq 1$. Since $\mathcal{E}$ is $\sigma$-strongly dense in $q M q \ominus M_{1} q$, for every $\varepsilon>0$, there exists $a \in \mathcal{E}$ such that $\|y-a\|_{\varphi} \leq \varepsilon / 2$. Thus, for every $i \in \mathcal{I}$, we have

$$
\left\|E_{M_{1}}\left(b^{*} w_{i}(y-a)\right)\right\|_{\varphi} \leq\left\|b^{*} w_{i}(y-a)\right\|_{\varphi} \leq\|y-a\|_{\varphi} \leq \varepsilon .
$$

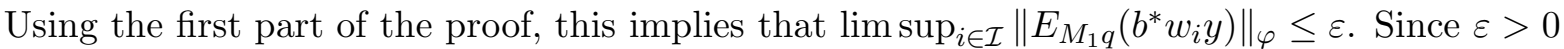
is arbitrary, we obtain $\lim _{i \in \mathcal{I}}\left\|E_{M_{1} q}\left(b^{*} w_{i} y\right)\right\|_{\varphi}=0$. This finishes the proof of the Claim.

Let $b \in \mathcal{E}$ and $y \in Q q \ominus M_{1} q$. Using the properties (P1) and (P2) of the net $\left(w_{i}\right)_{i \in \mathcal{I}}$, we obtain

$$
\begin{array}{rlr}
\left\|E_{M_{1} q}\left(b^{*} y\right)\right\|_{\varphi} & =\lim _{i \in \mathcal{I}}\left\|w_{i} E_{M_{1} q}\left(b^{*} y\right)\right\|_{\varphi} & \text { using }(\mathrm{P} 1) \text { for } a=E_{M_{1} q}\left(b^{*} y\right) \\
& =\lim _{i \in \mathcal{I}}\left\|E_{M_{1} q}\left(b^{*} y\right) w_{i}\right\|_{\varphi} & \text { using }(\mathrm{P} 2) \text { for } c=E_{M_{1} q}\left(b^{*} y\right) \\
& =\lim _{i \in \mathcal{I}}\left\|E_{M_{1} q}\left(b^{*} y w_{i}\right)\right\|_{\varphi} & \text { since } w_{i} \in M_{1} q \\
& =\lim _{i \in \mathcal{I}}\left\|E_{M_{1} q}\left(b^{*} w_{i} y\right)\right\|_{\varphi} & \text { using (P2) for } c=y \\
& =0 & \text { using item (2) of the Claim. }
\end{array}
$$

Since $\mathcal{E}$ is $\sigma$-strongly dense in $q M q \ominus M_{1} q$, we may choose a net $\left(b_{j}\right)_{j \in J}$ in $\mathcal{E}$ such that $b_{j}^{*} \rightarrow$ $y^{*} \sigma$-strongly as $j \rightarrow \infty$. Since $E_{M_{1} q}: q M q \rightarrow M_{1} q$ is $\sigma$-strongly continuous, we obtain that $E_{M_{1} q}\left(b_{j}^{*} y\right) \rightarrow E_{M_{1} q}\left(y^{*} y\right) \sigma$-strongly as $j \rightarrow \infty$ and hence $E_{M_{1} q}\left(y^{*} y\right)=0$. This implies that $y^{*} y=0$ and hence $y=0$. Since $y \in Q q \ominus M_{1} q$ is arbitrary, we derive that $M_{1} q=$ $Q q$. This contradicts the maximality of the projection $z \in Q^{\prime} \cap M$ and finishes the proof of Theorem A,

Proof of Corollary $\mathbb{B}$. Let $M_{1} \subset Q \subset M$ be any intermediate von Neumann subalgebra with faithful normal conditional expectation $E_{Q}: M \rightarrow Q$. Denote by $E_{M_{1}}: M \rightarrow M_{1}$ the unique $\varphi$-preserving normal conditional expectation. Since $M_{1}^{\varphi_{1}}$ is diffuse, we have $M_{1}^{\prime} \cap M \subset M_{1}$ by [Ue11, Corollary 3.2] and hence $E_{M_{1}}$ is the unique faithful normal conditional expectation from $M$ to $M_{1}$ by [Co72, Théorème 1.5.5]. Since $E_{M_{1}} \circ E_{Q}$ is a faithful normal conditional expectation from $M$ to $M_{1}$, we have $E_{M_{1}} \circ E_{Q}=E_{M_{1}}$. This implies that for every $x \in M$, we have

$$
\varphi\left(E_{Q}(x)\right)=\varphi\left(E_{M_{1}}\left(E_{Q}(x)\right)\right)=\varphi\left(\left(E_{M_{1}} \circ E_{Q}\right)(x)\right)=\varphi\left(E_{M_{1}}(x)\right)=\varphi(x) .
$$

By [Ta03, Theorem IX.4.2], we obtain that $Q$ is globally invariant under the modular automorphism group $\left(\sigma_{t}^{\varphi}\right)$.

Since $Q^{\prime} \cap M=\mathcal{Z}(Q)$ is abelian, there exists a sequence of pairwise orthogonal projections $q_{n} \in Q^{\prime} \cap M \subset \mathcal{Z}\left(M_{1}\right)$ such that $\sum_{n} q_{n}=1,\left(Q q_{0}\right)^{\prime} \cap q_{0} M q_{0}=\left(Q^{\prime} \cap M\right) q_{0}$ is a diffuse abelian von Neumann algebra and $Q q_{n}$ is a diffuse factor such that $\left(Q q_{n}\right)^{\prime} \cap q_{n} M q_{n}=\left(Q^{\prime} \cap M\right) q_{n}=\mathbf{C} q_{n}$ for every $n \geq 1$. Define

$$
\mathcal{I}=\{0\} \cup\left\{n \geq 1:\left(Q q_{n}\right)^{\prime} \cap\left(q_{n} M q_{n}\right)^{\omega} \text { is diffuse }\right\} .
$$

Put $z_{0}=\sum_{n \in \mathcal{I}} q_{n}$ and $N=\left(\mathbf{C} z_{0} \oplus M_{1} z_{0}^{\perp}\right) \vee M_{2}$. If $z_{0}=0$, then $M_{1} z_{0}=Q z_{0}$. Otherwise, by [Ue11, Lemma 2.2], we have that $M_{1} z_{0}$ and $z_{0} N z_{0}$ generate $z_{0} M z_{0}$ and are free in $z_{0} M z_{0}$ with respect to the state $\varphi_{z_{0}}=\frac{\varphi\left(z_{0} \cdot z_{0}\right)}{\varphi\left(z_{0}\right)}$. Thus, we have

$$
\left(z_{0} M z_{0}, \varphi_{z_{0}}\right)=\left(M_{1} z_{0}, \varphi_{z_{0}}\right) *\left(z_{0} N z_{0}, \varphi_{z_{0}}\right) .
$$

Moreover, the intermediate subalgebra $M_{1} z_{0} \subset Q z_{0} \subset z_{0} M z_{0}$ is globally invariant under the modular automorphism group $\left(\sigma_{t}^{\varphi z_{0}}\right)$ and we have

$$
\bigoplus_{n \in \mathcal{I}}\left(Q q_{n}\right)^{\prime} \cap\left(q_{n} M q_{n}\right)^{\omega} \subset\left(Q z_{0}\right)^{\prime} \cap\left(z_{0} M z_{0}\right)^{\omega} .
$$

Since $Q \subset M$ is globally invariant under the modular automorphism group $\left(\sigma_{t}^{\varphi}\right)$ and since $q_{n} \in M^{\varphi}$ for all $n \in \mathbf{N}$, we have that both $\bigoplus_{n \in \mathcal{I}}\left(Q q_{n}\right)^{\prime} \cap\left(q_{n} M q_{n}\right)^{\omega}$ and $\left(Q z_{0}\right)^{\prime} \cap\left(z_{0} M z_{0}\right)^{\omega}$ 
are globally invariant under the modular automorphism group $\left(\sigma_{t}^{\varphi_{z_{0}}^{\omega}}\right)$. Therefore, the inclusion (5) is with expectation. Since $\bigoplus_{n \in \mathcal{I}}\left(Q q_{n}\right)^{\prime} \cap\left(q_{n} M q_{n}\right)^{\omega}$ is diffuse, so is $\left(Q z_{0}\right)^{\prime} \cap\left(z_{0} M z_{0}\right)^{\omega}$ by Proposition 2.2. Applying Theorem $₫$ to the intermediate von Neumann subalgebra $M_{1} z_{0} \subset$ $Q z_{0} \subset z_{0} M z_{0}$, we obtain $M_{1} z_{0}=Q z_{0}$.

For every $n \notin \mathcal{I},\left(Q q_{n}\right)^{\prime} \cap\left(q_{n} M q_{n}\right)^{\omega}$ is not diffuse. By Proposition 2.5, we obtain that $\left(Q q_{n}\right)^{\prime} \cap$ $\left(q_{n} M q_{n}\right)^{\omega}=\mathbf{C} q_{n}$. In particular, since $Q \subset M$ is with expectation, we have $\left(Q q_{n}\right)^{\prime} \cap\left(Q q_{n}\right)^{\omega} \subset$ $\left(Q q_{n}\right)^{\prime} \cap\left(q_{n} M q_{n}\right)^{\omega}$. Thus, we have $\left(Q q_{n}\right)^{\prime} \cap\left(Q q_{n}\right)^{\omega}=\mathbf{C} q_{n}$ and so $Q q_{n}$ is a full nonamenable factor by Proposition 2.6. This finishes the proof of Corollary B.

Proof of Corollary $\square$. Let $M_{1}$ be any diffuse amenable von Neumann algebra with separable predual. Choose a faithful normal state $\varphi_{1}$ on $M_{1}$ such that the centralizer $M_{1}^{\varphi_{1}}$ is diffuse (see Proposition 2.2). Define $M_{2}=R_{\infty}$ to be the unique hyperfinite type $\mathrm{III}_{1}$ factor endowed with any faithful normal state $\varphi_{2}$. Then by [Ue11, Theorem 3.4], the free product $(M, \varphi)=\left(M_{1}, \varphi_{1}\right) *$ $\left(M_{2}, \varphi_{2}\right)$ is a full nonamenable type $\mathrm{III}_{1}$ factor. Moreover $M_{1} \subset M$ is with expectation.

Let $M_{1} \subset Q \subset M$ be any intermediate amenable von Neumann algebra with expectation. By Corollary $\mathrm{B}$, we obtain that $M_{1}=Q$.

4.2. Proof of Theorem D. We recall Popa's intertwining-by-bimodules theory that will play a crucial role in the proof of Theorem D, Let $M$ be a tracial von Neumann algebra together with $A \subset 1_{A} M 1_{A}$ and $B \subset 1_{B} M 1_{B}$ von Neumann subalgebras. Following [Po01, Po03], we say that $A$ embeds into $B$ inside $M$ and denote by $A \preceq_{M} B$ if one of the following equivalent conditions is satisfied:

- There exist projections $p \in A$ and $q \in B$, a nonzero partial isometry $v \in p M q$ and a unital normal $*$-homomorphism $\varphi: p A p \rightarrow q B q$ such that $a v=v \varphi(a)$ for all $a \in p A p$.

- There exist $\ell \geq 1$, a projection $q \in \mathbf{M}_{\ell}(B)$, a nonzero partial isometry $v \in \mathbf{M}_{1, \ell}\left(1_{A} M\right) q$ and a unital normal $*$-homomorphism $\varphi: A \rightarrow q \mathbf{M}_{\ell}(B) q$ such that $a v=v \varphi(a)$ for all $a \in A$.

- There is no net of unitaries $\left(w_{i}\right)_{i \in I}$ in $\mathcal{U}(A)$ such that $E_{B}\left(x^{*} w_{i} y\right) \rightarrow 0$ *-strongly as $i \rightarrow \infty$ for all $x, y \in p M q$.

We first prove the following intermediate result which can be regarded as a generalization of Theorem $\mathrm{A}$ in the case of tracial free product von Neumann algebras.

Theorem 4.1. Let $\left(M_{1}, \tau_{1}\right)$ and $\left(M_{2}, \tau_{2}\right)$ be von Neumann algebras with separable predual endowed with faithful normal tracial states. Assume that $M_{1}$ is diffuse. Denote by $(M, \tau)=$ $\left(M_{1}, \tau_{1}\right) *\left(M_{2}, \tau_{2}\right)$ the tracial free product von Neumann algebra.

For every von Neumann subalgebra $Q \subset M$ such that $Q \cap M_{1}$ and $Q^{\prime} \cap M^{\omega}$ are diffuse, we have $Q \subset M_{1}$.

Proof. Let $Q \subset M$ be any von Neumann subalgebra such that $Q \cap M_{1}$ and $Q^{\prime} \cap M^{\omega}$ are diffuse. By IPP05, Theorem 1.1], we have $Q^{\prime} \cap M \subset M_{1}$. Denote by $z \in \mathcal{Z}\left(Q^{\prime} \cap M\right)$ the maximum projection such that $Q z \subset z M_{1} z$. We prove that $z=1$. Assume by contradiction that this not the case and put $q=z^{\perp}=1-z \in \mathcal{Z}\left(Q^{\prime} \cap M\right) \subset M_{1}$. We have $q \neq 0$.

First, assume that $Q q$ is amenable. Choose a diffuse abelian subalgebra $A \subset q^{\perp} M_{1} q^{\perp}$ and put $\mathcal{Q}=Q q \oplus A$. Then $\mathcal{Q}$ is amenable and $\mathcal{Q} \cap M_{1}$ is diffuse. Theorem 3.1 implies that the inclusion $M_{1} \subset M$ has the asymptotic orthogonality property relative to the diffuse subalgebra $\mathcal{Q} \cap M_{1}$ in the sense of [Ho12b, Definition 5.1]. Since the inclusion $M_{1} \subset M$ is mixing (see e.g. [Ho12b, Proposition 4.7]) in the sense of [Ho12b, Definition 4.4], we have that the inclusion $M_{1} \subset M$ is weakly mixing through the diffuse subalgebra $\mathcal{Q} \cap M_{1}$ in the sense of [Ho12b, Definition 4.1]. Therefore [Ho12b, Theorem 8.1] implies that $\mathcal{Q} \subset M_{1}$ and so $Q q \subset q M_{1} q$. This contradicts the fact that $z$ is the maximum projection in $\mathcal{Z}\left(Q^{\prime} \cap M\right)$ such that $Q z \subset z M_{1} z$. 
Second, assume that $Q q$ is not amenable. Let $q_{0} \in \mathcal{Z}\left(Q^{\prime} \cap M\right) q$ be a nonzero central projection such that $Q q q_{0}$ has no amenable direct summand. Since $\left(Q q q_{0}\right)^{\prime} \cap\left(q q_{0} M q q_{0}\right)^{\omega}=q q_{0}\left(Q^{\prime} \cap\right.$ $\left.M^{\omega}\right) q q_{0}$ is diffuse and since the inclusion $M_{1} \subset M$ is mixing, by $\mathrm{Pe} 06$, Theorems 4.3, 4.5 and Lemma 5.1] and [IPP05, Theorem 4.3] (see also [Ho07, Theorem 5.6] and [Io12, Theorem 6.3]), we obtain that $Q q q_{0} \preceq_{M} M_{i}$ for some $i \in\{1,2\}$. This implies that $Q q \preceq_{M} M_{i}$ for some $i \in\{1,2\}$.

There exist $\ell \geq 1$, a projection $p \in \mathbf{M}_{\ell}\left(M_{i}\right)$, a nonzero partial isometry $v \in \mathbf{M}_{1, \ell}(q M) p$ and a unital normal $*$-homomorphism $\varphi: Q q \rightarrow p \mathbf{M}_{\ell}\left(M_{i}\right) p$ such that $a v=v \varphi(a)$ for all $a \in Q q$. Write $v=\left[v_{1} \cdots v_{\ell}\right] \in \mathbf{M}_{1, \ell}(q M) p$. In particular, we have $Q v_{j} \subset \sum_{k=1}^{\ell} v_{k} M_{i}$ for all $1 \leq j \leq \ell$ and so $\left(Q \cap M_{1}\right) v_{j} \subset \sum_{k=1}^{\ell} v_{k} M_{i}$ for all $1 \leq j \leq \ell$. Since $Q \cap M_{1}$ is diffuse, by [PP05, Theorem 1.1], we obtain that $i=1$ and that $v_{j} \in M_{1}$ for all $1 \leq j \leq \ell$. Therefore $v v^{*} \in(Q q)^{\prime} \cap q M_{1} q$ is a nonzero projection such that $Q v v^{*} \subset v v^{*} M_{1} v v^{*}$. If we denote by $z_{0}$ the central support of $v v^{*}$ in $(Q q)^{\prime} \cap q M_{1} q$, we have that $z_{0} \in \mathcal{Z}\left(Q^{\prime} \cap M\right) q, z_{0} \neq 0$ and $Q z_{0} \subset z_{0} M_{1} z_{0}$. This contradicts again the fact that $z$ is the maximum projection in $\mathcal{Z}\left(Q^{\prime} \cap M\right)$ such that $Q z \subset z M_{1} z$.

Consequently, we obtain that $z=1$ and so $Q \subset M_{1}$. This finishes the proof of Theorem4.1,

Proof of Theorem $D$. The proof is similar to the one of Corollary $\mathbb{B}$, Let $Q \subset M$ be any von Neumann subalgebra such that $Q \cap M_{1}$ is diffuse. By [Io12, Lemma 2.7], there exists a central projection $z \in \mathcal{Z}\left(Q^{\prime} \cap M\right) \cap \mathcal{Z}\left(Q^{\prime} \cap M^{\omega}\right) \subset M_{1}$ such that $\left(Q^{\prime} \cap M^{\omega}\right) z$ is diffuse and $\left(Q^{\prime} \cap M^{\omega}\right) z=\left(Q^{\prime} \cap M\right) z$ is discrete. Choose a diffuse abelian subalgebra $A \subset z^{\perp} M_{1} z^{\perp}$ and put $\mathcal{Q}=Q z \oplus A$. We have that $\mathcal{Q} \cap M_{1}$ and $\mathcal{Q}^{\prime} \cap M^{\omega}$ are diffuse. By Theorem 4.1, we obtain $\mathcal{Q} \subset M_{1}$ and hence $Q z \subset z M_{1} z$. This finishes the proof of Theorem D.

\section{REFERENCES}

[AH12] H. Ando, U. HaAgerup, Ultraproducts of von Neumann algebras. J. Funct. Anal. 266 (2014), 6842-6913.

[Ba93] L. BARnett, Free product von Neumann algebras of type III. Proc. Amer. Math. Soc. 123 (1995), $543-553$.

[BC13] R. Boutonnet, A. CARderi, Maximal amenable subalgebras of von Neumann algebras associated with hyperbolic groups. arXiv:1310.5864

[Br12] A. BRothiER, The cup subalgebra of a $\mathrm{II}_{1}$ factor given by a subfactor planar algebra is maximal amenable. Pacific J. Math., to appear. arXiv:1210.8091

[CFRW08] J. Cameron, J. Fang, M. Ravichandran, S. White, The radial masa in a free group factor is maximal injective. J. Lond. Math. Soc. 82 (2010), 787-809.

[Co72] A. Connes, Une classification des facteurs de type III. Ann. Sci. École Norm. Sup. 6 (1973), $133-252$.

[Co74] A. Connes, Almost periodic states and factors of type $\mathrm{III}_{1}$. J. Funct. Anal. 16 (1974), 415-445.

[Co75a] A. Connes, Outer conjugacy classes of automorphisms of factors. Ann. Sci. École Norm. Sup. 8 (1975), 383-419.

[Co75b] A. Connes, Classification of injective factors. Ann. of Math. 104 (1976), 73-115.

[Co85] A. Connes, Factors of type $\mathrm{III}_{1}$, property $\mathrm{L}_{\lambda}^{\prime}$ and closure of inner automorphisms. J. Operator Theory 14 (1985), 189-211.

[Dy92] K. Dykema, Factoriality and Connes' invariant $T(\mathcal{M})$ for free products of von Neumann algebras. J. reine angew. Math. 450 (1994), 159-180.

[Fa06] J. FANG, On maximal injective subalgebras of tensor products of von Neumann algebras. J. Funct. Anal. 244 (2007), 277-288.

[Ga09] M. GaO, On maximal injective subalgebras. Proc. Amer. Math. Soc. 138 (2010), 2065-2070.

[Ge95] L. GE, On maximal injective subalgebras of factors. Adv. Math. 118 (1996), 34-70.

[Ha84] U. HAAGERup, Connes' bicentralizer problem and uniqueness of the injective factor of type $\mathrm{III}_{1}$. Acta Math. 158 (1987), 95-148.

[Ho07] C. Houdayer, Construction of type $\mathrm{II}_{1}$ factors with prescribed countable fundamental group. J. Reine Angew. Math. 634 (2009), 169-207.

[Ho12a] C. Houdayer, A class of $\mathrm{II}_{1}$ factors with an exotic abelian maximal amenable subalgebra. Trans. Amer. Math. Soc. 366 (2014), 3693-3707.

[Ho12b] C. HoudAYER, Structure of $\mathrm{II}_{1}$ factors arising from free Bogoljubov actions of arbitrary groups. Adv. Math. 260 (2014), 414-457. 
[HS90] U. HaAgerup, E. Størmer, Equivalence of normal states on von Neumann algebras and the flow of weights. Adv. Math. 83 (1990), 180-262.

[Io12] A. IoAnA, Cartan subalgebras of amalgamated free product $\mathrm{II}_{1}$ factors. Ann. Sci. École Norm. Sup., to appear. arXiv: 1207.0054

[IPP05] A. IoAna, J. Peterson, S. Popa, Amalgamated free products of w-rigid factors and calculation of their symmetry groups. Acta Math. 200 (2008), 85-153.

[Jo10] P. Jolissaint, Maximal injective and mixing masas in group factors. arXiv:1004.0128

[MvN43] F. Murray, J. von Neumann, Rings of operators. IV. Ann. of Math. 44 (1943), 716-808.

[Oc85] A. OCNEANU, Actions of discrete amenable groups on von Neumann algebras. Lecture Notes in Mathematics, 1138. Springer-Verlag, Berlin, 1985. iv+115 pp.

[Pe06] J. Peterson, L L ${ }^{2}$-rigidity in von Neumann algebras. Invent. Math. 175 (2009), 417-433.

[Po83] S. PopA, Maximal injective subalgebras in factors associated with free groups. Adv. Math. 50 (1983), $27-48$.

[Po01] S. PopA, On a class of type $\mathrm{II}_{1}$ factors with Betti numbers invariants. Ann. of Math. 163 (2006), 809-899.

[Po03] S. PopA, Strong rigidity of $\mathrm{II}_{1}$ factors arising from malleable actions of w-rigid groups I and II. Invent. Math. 165 (2006), 369-408, 409-451.

[Po13] S. PopA, A II 1 factor approach to the Kadison-Singer problem. Comm. Math. Phys. 332 (2014), 379-414.

[Sh05] J. Shen, Maximal injective subalgebras of tensor products of free group factors. J. Funct. Anal. 240 (2006), 334-348.

[Ta03] M. TAKESAKI, Theory of operator algebras. II. Encyclopaedia of Mathematical Sciences, 125. Operator Algebras and Non-commutative Geometry, 6. Springer-Verlag, Berlin, 2003. xxii+518 pp.

[Ue98] Y. UEDA, Amalgamated free products over Cartan subalgebra. Pacific J. Math. 191 (1999), 359-392.

[Ue11] Y. UEDA, Factoriality, type classification and fullness for free product von Neumann algebras. Adv. Math. 228 (2011), 2647-2671.

[Vo85] D.-V. Voiculescu, Symmetries of some reduced free product $C^{*}$-algebras. Operator algebras and Their Connections with Topology and Ergodic Theory, Lecture Notes in Mathematics 1132. SpringerVerlag, (1985), 556-588.

[Vo92] D.-V. Voiculescu, K.J. Dykema, A. NicA, Free random variables. CRM Monograph Series 1. American Mathematical Society, Providence, RI, 1992.

CNRS - Université Paris-Est - Marne-la-Vallée, LAMA UMR 8050, 77454 Marne-la-Vallée Cedex 2, FRANCE

E-mail address: cyril.houdayer@u-pem.fr 